Delft University of Technology

\title{
A Decentralized Event-Based Approach for Robust Model Predictive Control
}

Kolarijani, Arman Sharifi; Bregman, Sander Christian; Esfahani, Peyman Mohajerin; Keviczky, Tamas

DOI

10.1109/TAC.2019.2945285

Publication date

2020

Document Version

Final published version

Published in

IEEE Transactions on Automatic Control

\section{Citation (APA)}

Kolarijani, A. S., Bregman, S. C., Esfahani, P. M., \& Keviczky, T. (2020). A Decentralized Event-Based Approach for Robust Model Predictive Control. IEEE Transactions on Automatic Control, 65(8), 3517-3529. https://doi.org/10.1109/TAC.2019.2945285

\section{Important note}

To cite this publication, please use the final published version (if applicable).

Please check the document version above.

\section{Copyright}

Other than for strictly personal use, it is not permitted to download, forward or distribute the text or part of it, without the consent of the author(s) and/or copyright holder(s), unless the work is under an open content license such as Creative Commons.

\section{Takedown policy}

Please contact us and provide details if you believe this document breaches copyrights.

We will remove access to the work immediately and investigate your claim. 
Green Open Access added to TU Delft Institutional Repository

'You share, we take care!' - Taverne project

https://www.openaccess.nl/en/you-share-we-take-care

Otherwise as indicated in the copyright section: the publisher is the copyright holder of this work and the author uses the Dutch legislation to make this work public. 


\title{
A Decentralized Event-Based Approach for Robust Model Predictive Control
}

\author{
Arman Sharifi Kolarijani ${ }^{\circledR}$, Sander Christian Bregman (), Peyman Mohajerin Esfahani ${ }^{\circledR}$, \\ and Tamás Keviczky (i)
}

\begin{abstract}
In this paper, we propose an event-based sampling policy to implement a constraint-tightening, robust MPC method. The proposed policy enjoys a computationally tractable design and is applicable to perturbed, linear time-invariant systems with polytopic constraints. In particular, the triggering mechanism is suitable for plants with no centralized sensory node as the triggering mechanism can be evaluated locally at each individual sensor. From a geometrical viewpoint, the mechanism is a sequence of hyperrectangles surrounding the optimal state trajectory such that robust recursive feasibility and robust stability are guaranteed. The design of the triggering mechanism is cast as a constrained parametric-in-set optimization problem with the volume of the set as the objective function. Reparameterized in terms of the set vertices, we show that the problem admits a finite tractable convex program reformulation and a linear program relaxation. Several numerical examples are presented to demonstrate the effectiveness and limitations of the theoretical results.
\end{abstract}

Index Terms-Convex optimization, event-triggered control, polytope, robust model predictive control (RMPC).

\section{INTRODUCTION}

$\mathbf{N}$ OWADAYS, networked control systems (NCSs) generally demand an array of compatibility and efficiency measures from control design methods, such as utilization under shared resources, applicability to mobile tasks, and compatibility with digital communication infrastructures [1]. Event-based control (EBC) is a class of strategies that aims to improve the efficiency of NCSs in the context of communication and computation. In EBC, the dynamics determine the instance to update a control action (contrary to the traditional case where a control action is updated periodically) [2]. There are two options to implement such an event-based logic_embedded in the sensory system, the so-called event-triggered control [3], [4], or embedded in the controller, the so-called self-triggered control [5], [6]. The responsible entity to determine an update

Manuscript received July 9, 2019; revised September 21, 2019; accepted September 28, 2019. Date of publication October 2, 2019; date of current version July 28, 2020. Recommended by Associate Editor E. C. Kerrigan. (Corresponding author: Arman Sharifi Kolarijani.)

The authors are with the Delft Center for Systems and Control, Delft University of Technology, 2628 CD Delft, The Netherlands (email: arman.sh.kolarigani@gmail.com; s.c.bregman@student.tudelft.nl; p.mohajerin@gmail.com; t.keviczky@tudelft.nl).

Color versions of one or more of the figures in this article are available online at http://ieeexplore.ieee.org.

Digital Object Identifier 10.1109/TAC.2019.2945285 instance is known as the triggering mechanism. In particular, model predictive control (MPC) methods [7] have been the subject of many studies in order to be amended with an EBC mindset.

MPC methods are a class of on-line optimization-based control approaches. In these methods, a measure of system performance is optimized over a finite horizon while states and inputs are subject to certain constraints. When the underlying dynamics is uncertain, the specific term robust MPC (RMPC) is used for these methods in the literature [8]. We refer the interested reader to the survey papers [9] and [10] that discuss about different aspects of MPC.

Traditionally, the controller solves the corresponding optimization problem at every time step and produces as outcomes two sequences of optimal inputs and states. Then, the controller sends the first element of input sequence to the actuators and the remaining elements of the input sequence and the whole state sequence are discarded. These discarded predictions in a standard MPC setting can serve as a basis to design a triggering mechanism. Moreover, the computational burden of MPC methods is a major drawback, hindering their usage in practice. One thus hopes, by employing an EBC approach, to reduce the frequency at which the underlying optimization problem is solved. Notice that there are already some techniques in the MPC literature (the so-called warm start approaches [11]) that exploit the computed sequences at the previous step to speed up the computation process.

There is also a big incentive to exploit the computed sequences of MPC methods in a class of NCSs, namely, wireless sensor/actuator networks (WSANs). In these systems, the most important concern is the energy efficiency, see, e.g., [12, Sec. IV-B]. The main source of energy depletion in a wireless node is the transceiver (responsible for sending and receiving data). To reduce the frequency of data transfer, it is, hence, more efficient (energywise) to aggregate the data into a single packet (if possible) and transmit the resulting packet at once over the communication network [13] and [14].

Statement of contribution: In this paper, an event-triggered (ET) approach is proposed to implement an RMPC method on perturbed, linear time-invariant (LTI) systems. The RMPC method is originally introduced in [15]. The core idea behind the ET approach is to construct a sequence of hyperrectangles around the optimal state sequence available from solving the RMPC problem. Then, these hyperrectangles will be sent to the sensors. The optimal input sequence will also be transmitted 
to the actuators. Once the observed states at the sensory units leave these hyperrectangles, a triggering happens and the states at the triggering instance will be transmitted to the controller. This procedure is then repeated in a sampled-data fashion. A key feature of the proposed ET approach is its ability to decide, based on the local observation of each individual sensor, whether to trigger or not. This feature stems from the fact that the sets describing the triggering mechanism are hyperrectangles. Hence, the conditions required for a triggering in different states of the system are independent of each other. This feature is particularly appealing to systems with decentralized (spatially dispersed) sensing units, including systems equipped with high-level (or supervisory) MPC methods, e.g., water treatment systems [16], HVAC systems [17], and commercial refrigeration systems [18] to name a few. The collocation of the triggering mechanism and the sensory units is physically impossible in such systems. Moreover, the addition of a central node (on which the triggering mechanism is placed on) to collect the sensory data comes at the price of extra communication bandwidth usage. On the theoretical side, the design of the ET approach is decoupled from the design of the underlying RMPC method. As a result, a fair comparison between the performances of the ET and standard implementations of the RMPC method becomes possible. This paper extends the results of the authors' previous work in [19] in multiple directions, in particular, by simplifying the triggering "law." The approach in [19] requires an "advanced" triggering mechanism that is responsible for: 1) constructing certain input and state sequences; 2) evaluating the satisfaction of MPC's constraints by these sequences; and 3 ) comparing the values of the cost function based on the constructed sequences with the value function at the last triggering instance. The main contributions of this paper are summarized as follows.

1) Decoupled recursive feasibility and stability: Given an RMPC method in place, we propose a set-theory-based, ET approach that preserves robust recursive feasibility and robust stability. The proposed approach is decoupled from the control synthesis process and does not require additional assumptions, such as extra conditions on eigenvalues of weighting matrices in the cost function or the need to define user-specified thresholds for the triggering mechanism (see Theorem IV.1).

2) Decentralized applicability: The proposed approach enjoys a decentralized triggering mechanism that only requires local sensory information (see Definition III.3).

3) Tractable convex program reformulation: We show that a certain type of nonconvex volume-maximization problem with set-based constraints, that is deployed to design the triggering mechanism, admits a finite tractable convex program (CP) reformulation (see Theorem IV.4).

4) Suboptimal linear program (LP) relaxation: Motivated by an approach in the literature, we further show that an $\mathrm{LP}$ relaxation of the $\mathrm{CP}$ reformulation is possible (see Theorem IV.5).

Literature review: In what follows, we first review several event-triggered, MPC approaches. We then close this section by giving a brief account of several computationally efficient approaches that are customized for MPC problems. Related works: Let us first mention the shared properties of the references that follow-linear discrete-time models, event-triggering mechanisms, constrained MPC methods, minimal (to none) coupling of the parameters of the triggering mechanism and the considered MPC method, and a computationally viable approach to design the triggering mechanism.

To deal with practical issues, such as a band-limited communication channel, a novel design approach for NCSs is proposed in [20]. They employ the notion of moving horizon [21] to design the estimator and controller. A remarkable character of their approach is its ability to decide on the fly which input channel should be updated (i.e., a certain type input-channel event-triggering control). In case of collocated controller and actuator units, an event-based estimator with a bounded covariance matrix is designed in [22]. While the estimator receives data via a Lebesgue sampling approach, it periodically updates the controller's information regarding the disturbances with a polytopic overapproximation of the covariance matrix. The authors of [23] propose an interesting transmission strategy for wireless sensor/controller communications with practical energy-aware provisions (the controller is collocated with the actuator system). Using some predefined thresholds for each state's sensor (i.e., an $\ell_{1}$-type triggering mechanism), the controller is computed offline using an explicit MPC approach [24]. Based on a prescribed 2-norm ball around the optimal state trajectory, the authors in [25] propose a triggering mechanism for WSANs. They show that the approach is robustly stable to a set that is a function of the radius of threshold ball and the maximal 2-norm of disturbance. For linear, continuous-time dynamical systems affected by a Wiener process, a codesign method (i.e., simultaneous design of the scheduler and the controller) is proposed in [26]. The main idea is inspired by the notion of rollout from dynamic programming [27]. More importantly, the authors show that under some mild conditions, an EBC approach outperforms a traditional control approach w.r.t. closed-loop performance/average transmission rate. (Notice that for most of the approaches in the literature including our paper such a guarantee is not provided.) A set theoretic triggering mechanism is introduced in [28] for systems with collocated controller and sensory units. The approach is inspired by the tube-based MPC proposed in [29]. By exploiting the known probability distribution of disturbance, they also guarantee an average sampling rate. However, their tube-contraction method requires a certain type of realization of a discrete-time system, see [28, Remark 8]. Demirel et al., introduce a sensor/actuator event-triggering mechanism for control systems with limited number of control messages (i.e., communication and computation resources are scarce) [30]. They relax the underlying combinatorial problem into a convex one by an appropriate definition of event thresholds. In [31], a packetized approach is proposed for inputaffine, nonlinear systems with bounded additive disturbances in continuous-time. In the proposed approach, an RMPC controller (connected via a communication network to the plant) takes into account the mismatched uncertainties while an integral sliding-mode controller [32] (placed at the plant) counters the effect of the matched uncertainties. 
Algorithmic viewpoint: An MPC optimization problem is computationally expensive by itself. Hence, the merit of an event-based policy of implementation would be lost if the mechanism demands a drastically higher computational effort compared to the underlying MPC problem. Dunn and Bertsekas in [33] exploit the structure of their problem to reduce the cubic complexity of computing a Newton step to a linear one. In [34], the authors use a specific ordering of decision variables to promote a sparse structure that decreases the cost of computing a control action. The authors in [35] employ a simple, gradientbased algorithm to solve an MPC problem while providing $a$ priori computational complexity certificate.

The layout of the paper is as follows. The mathematical notions used in the paper are outlined in Section II. Section III is devoted to the considered RMPC method. The main results regarding the event-based implementation policy are introduced in Section IV. Section V contains the technical proofs. Several numerical examples are presented in Section VI to evaluate the effectiveness and limitations of the theoretical results. Finally, we present several future research directions in Section VII.

\section{Notation And Preliminaries}

In what follows, we begin with a brief review of the mathematical preliminaries employed in the rest of the paper.

Notation: The set of nonnegative integers is denoted by $\mathbb{Z}_{>0}$. Given positive integers $m$ and $n, \mathbb{R}^{m}$ and $\mathbb{R}^{m \times n}$ represent the $m$-dimensional Euclidean space and the space of $m \times n$ matrices with real entries, respectively. Given two integers $i, j$ where $i \leq j,\{i: j\}:=\{i, i+1, \ldots, j\}$. For any pairs of vectors $a, b \in \mathbb{R}^{n}$, the inequality $a<(\leq) b$ is realized in a componentwise manner. Given a vector $v \in \mathbb{R}^{n}$ and a scalar $p \geq 1,\|v\|_{p}$ denotes the $p$-norm $\left(\sum_{i=1}^{n}\left(v^{i}\right)^{p}\right)^{1 / p}$. Given a matrix $M \in \mathbb{R}^{m \times n}, M_{i j}$ denotes the $i$ th row, $j$ th column entry of $M$. Moreover, the matrix $M^{+} \in \mathbb{R}^{m \times n}$ is the matrix with entries $M_{i j}^{+}:=\max \left\{0, M_{i j}\right\}$. The $n \times n$ zero and identity matrices are denoted by $0_{n}$ and $\mathrm{I}_{n}$, respectively. Given a set $\mathcal{S} \subset \mathbb{R}^{n}$ and a matrix $M \in \mathbb{R}^{m \times n}$, the set $M \mathcal{S}$ denotes the set $\{c \in$ $\left.\mathbb{R}^{m}: \exists s \in \mathcal{S}, M s=c\right\}$. Given a matrix $M \succ 0$ (i.e., positive definite), the squared weighted distance of a point $r \in \mathbb{R}^{n}$ from a closed set $\mathcal{S} \subset \mathbb{R}^{n}$ is defined as $d_{M}(r, \mathcal{S}):=\min _{s \in \mathcal{S}} \| r-$ $s \|_{M}^{2}=\min _{s \in \mathcal{S}}(r-s)^{\top} M(r-s)$. Denote the projection of $r$ onto $\mathcal{S}$ by $\Pi_{M}(r, \mathcal{S}) \in \operatorname{argmin}_{s \in \mathcal{S}} d_{M}(r, \mathcal{S})$. Note that when $\mathcal{S}$ is also convex, the projection is unique. Given sets $\mathcal{C}$ and $\mathcal{D}$, the Pontryagin difference $\mathcal{C} \ominus \mathcal{D}$ and the Minkowski sum $\mathcal{C} \oplus \mathcal{D}$ are defined as $\mathcal{C} \ominus \mathcal{D}:=\{c: c+d \in \mathcal{C}, \forall d \in \mathcal{D}\}$ and $\mathcal{C} \oplus \mathcal{D}:=\{c+d: \forall c \in \mathcal{C}, \forall d \in \mathcal{D}\}$, respectively. The function $\operatorname{sign}(\cdot)$ represents the standard sign function. Given a set $\mathcal{X} \in$ $\mathbb{R}^{n}$ and an extended real-valued function $f: \mathcal{X} \rightarrow[-\infty,+\infty]$, the effective domain of $f$ is the set $\operatorname{dom}(f)=\{x \in \mathcal{X}: f(x)<$ $\infty\}$.

The following result will be used frequently in the development of the triggering mechanism.

Lemma II.1 (Set-difference lower bound [15]): Let $r$ be a vector in $\mathbb{R}^{n}, \mathcal{B}$ and $\mathcal{C}$ be two compact sets in $\mathbb{R}^{n}$, and $M$ be a positive definite matrix in $\mathbb{R}^{n \times n}$. Then, $d_{M}(r+c, \mathcal{B}) \leq$ $d_{M}(r, \mathcal{B} \ominus \mathcal{C})$, for all $c \in \mathcal{C}$.
We now revisit some notions from convex analysis (see e.g., [36, Sec. 2] for a compact exposition of the subject). Given a set $\mathcal{S} \subset \mathbb{R}^{n}$, the support function of $\mathcal{S}$ evaluated at $\eta \in \mathbb{R}^{n}$ is $h_{\mathcal{S}}(\eta):=\sup _{s \in \mathcal{S}}\langle\eta, s\rangle$. The domain $\mathcal{K}_{\mathcal{S}}$ on which the support function is defined is a convex cone pointed at the origin. If $\mathcal{S}$ is bounded, then $\mathcal{K}_{\mathcal{S}}:=\mathbb{R}^{n}$. Given a matrix $M \in \mathbb{R}^{n \times m}$ and a vector $v \in \mathbb{R}^{n}$, if $M^{\top} v \in \mathcal{K}_{\mathcal{S}}$, then $h_{M \mathcal{S}}(v):=h_{\mathcal{S}}\left(M^{\top} v\right)$. Suppose $\mathcal{S} \subset \mathbb{R}^{n}$ is closed and convex. Then, $\mathcal{S}:=\left\{s \in \mathbb{R}^{n}\right.$ : $\left.\langle\eta, s\rangle \leq h_{\mathcal{S}}(\eta), \forall \eta \in \mathcal{K}_{\mathcal{S}}\right\}$, i.e., the intersection of its supporting half-planes. A set $\mathcal{S} \subset \mathbb{R}^{n}$ is called a polyhedron, if $\mathcal{S}=\{s \in$ $\left.\mathbb{R}^{n}: A_{\mathcal{S}} s \leq b_{\mathcal{S}}\right\}, A_{\mathcal{S}} \in \mathbb{R}^{m \times n}, b_{\mathcal{S}} \in \mathbb{R}^{m}$. If the polyhedron $\mathcal{S}$ is bounded, the set is called a polytope and its representation given above is known as the $H$-representation. Furthermore, the support function $h_{\mathcal{S}}(\eta)$ of a polytope $\mathcal{S}$ is the solution of the LP, $h_{\mathcal{S}}(\eta)=\max _{s}\langle\eta, s\rangle$ subject to $A_{\mathcal{S}} s \leq b_{\mathcal{S}}$. Given the $H$-representation of a polytope, we employ the notations $a_{i, \mathcal{S}} \in \mathbb{R}^{1 \times n}$ and $a_{\mathcal{S}, j} \in \mathbb{R}^{m \times 1}$ to denote the $i$ th row and the $j$ th column of $A_{\mathcal{S}}$, respectively. Moreover, $b_{i, \mathcal{S}}$ is the $i$ th entry of $b_{\mathcal{S}}$. Given a polyhedron $\mathcal{S} \subset \mathbb{R}^{n}$ and a set $\mathcal{V} \subset \mathbb{R}^{n}$, assume that $h_{\mathcal{V}}\left(a_{i, \mathcal{S}}^{\top}\right)$ is well defined for all $i \in\{1: m\}$. Then, $\mathcal{S} \ominus \mathcal{V}:=$ $\left\{z \in \mathbb{R}^{n}:\left\langle a_{i, \mathcal{S}}^{\top}, z\right\rangle \leq b_{i, \mathcal{S}}-h_{\mathcal{V}}\left(a_{i, \mathcal{S}}^{\top}\right), \forall i \in\{1: m\}\right\}$. For any vector-pairs $l, u \in \mathbb{R}^{n}$ such that $l<u$, the full-dimensional convex polytope $\mathcal{B}(l, u):=\left\{x \in \mathbb{R}^{n}: l \leq x \leq u\right\}=\left\{x \in \mathbb{R}^{n}\right.$ : $\left.A_{\mathcal{B}} x \leq b_{\mathcal{B}}\right\}$ is called a hyperrectangle, where $A_{\mathcal{B}}:=\left[\mathrm{I}_{n}-\mathrm{I}_{n}\right]^{\top}$ and $b_{\mathcal{B}}=\left[u^{\top}-l^{\top}\right]^{\top}$.

\section{Robust Model Predictive Control Method}

In this section, we introduce the class of constrained dynamical systems considered in this paper, followed by the description of the RMPC method. At last, we formally state the problem addressed in this paper.

Consider an LTI system with a bounded additive disturbance given by

$$
x^{+}=A x+B u+w
$$

where $x^{+}$is the successor state and $x, u$, and $w$ are the current state, input, and disturbance, respectively. The current state, input, and disturbance are subject to the hard constraints

$$
x \in \mathbb{X} \subset \mathbb{R}^{n_{x}} \quad u \in \mathbb{U} \subset \mathbb{R}^{n_{u}} \quad w \in \mathbb{W} \subset \mathbb{R}^{n_{x}} .
$$

A system is called the nominal system associated with (1) when $w=0$. Given a positive integer $N$, let $\mathscr{U}:=\mathbb{U}^{N}=\prod_{i=0}^{N-1} \mathbb{U}$ $\left(\mathscr{W}:=\mathbb{W}^{N}\right)$ denote the class of admissible control sequences $\boldsymbol{u}:=\left\{u_{i}\right\}_{i \in\{0: N-1\}}$ (admissible disturbance sequences $\boldsymbol{w}:=$ $\left.\left\{w_{i}\right\}_{i \in\{0: N-1\}}\right)$. Initiated at state $x$, the solution to (1) at time $i$ with the control and disturbance sequences $\boldsymbol{u}$ and $\boldsymbol{w}$, respectively, is denoted by $\phi_{i}^{\boldsymbol{u}, \boldsymbol{w}}(x)$. Similarly, we define $\boldsymbol{\phi}^{\boldsymbol{u}, \boldsymbol{w}}(x):=$ $\left\{\phi_{i}^{\boldsymbol{u}, \boldsymbol{w}}(x)\right\}_{i \in\{0: N\}}$. Moreover, let $\phi_{i}^{\boldsymbol{u}, \boldsymbol{0}}(x)$ denote the nominal solution with the input sequence $\boldsymbol{u}$ initiated at state $x$. The RMPC method is designed such that the state $x$ and the input $u$ eventually converge to some user-defined target sets $\mathbb{T}^{\mathbb{X}} \subset \mathbb{R}^{n_{x}}$ and $\mathbb{T}^{\mathbb{U}} \subset \mathbb{R}^{n_{u}}$, respectively, while the constraints (2) are satisfied at all times.

Assumption III.1 (System and constraint sets): 1) Nominal controllability: The pair $(A, B)$ is controllable. 2) Polytopic sets: The sets $\mathbb{X}, \mathbb{U}, \mathbb{T}^{\mathbb{X}}, \mathbb{T}^{\mathbb{U}}$, and $\mathbb{W}$ are all convex, compact 
polytopes containing their underlying spaces' origin in their interior.

We start by introducing two types of feedback gains which are used in the RMPC method and are essential for the construction of the triggering mechanism. Let $F \in \mathbb{R}^{n \times m}$ be a given feedback gain that guarantees the stability of the nominal system with $u=$ $F x$. The nominal gain $F$ can be designed so that a satisfactory performance (e.g., in an LQ optimal control sense) is guaranteed for the nominal system.

Let integer $N \geq n_{x}+1$ be the horizon length of the RMPC method and integer $M$ be given, where $M \in\left\{n_{x}: N-1\right\}$. Suppose next that a set of feedback gains $\boldsymbol{K}=\left\{K_{i}\right\}_{i \in\{0: N-1\}}$ are given such that $\prod_{i=1}^{M}\left(A+B K_{i}\right)=0$, i.e., for all $k \geq M$, $\phi_{k}^{\boldsymbol{u}, \mathbf{0}}(x)=0$. We call the set of gains $\boldsymbol{K}$ the tightening gains since these gains are employed in the state and input constraint tightening process. We refer the interested reader to [15, Sec. IV] for a possible approach to construct the gains $\boldsymbol{K}$. The constraint tightening approach is applied to the input, state, input target, and state target sets, that is, for all $i \in\{0: N-2\}$

$$
\begin{aligned}
\mathcal{U}_{0} & =\mathbb{U}, & \mathcal{U}_{i+1} & =\mathcal{U}_{i} \ominus K_{i} L_{i} \mathbb{W} \\
\mathcal{X}_{0} & =\mathbb{X}, & \mathcal{X}_{i+1} & =\mathcal{X}_{i} \ominus L_{i} \mathbb{W} \\
\mathcal{T}_{0}^{\mathbb{U}} & =\mathbb{T}^{\mathbb{U},} & \mathcal{T}_{i+1}^{\mathbb{U}} & =\mathcal{T}_{i}^{\mathbb{U}} \ominus K_{i} L_{i} \mathbb{W} \\
\mathcal{T}_{0}^{\mathbb{X}} & =\mathbb{T}^{\mathbb{X},} & \mathcal{T}_{i+1}^{\mathbb{X}} & =\mathcal{T}_{i}^{\mathbb{X}} \ominus L_{i} \mathbb{W}
\end{aligned}
$$

where $L_{0}=\mathrm{I}_{n_{x}}$ and $L_{i+1}=\left(A+B K_{i}\right) L_{i}$ for all $i \in\{0: N-$ $2\}$. The $M$-step nilpotency of the set of gains $\mathbf{K}$ implies that for all $i \in\{M: N-1\}, L_{i}=0_{n_{x}}$.

Let the terminal set $\mathcal{X}_{f} \subset \mathbb{R}^{n_{x}}$ be a control invariant set for the nominal system, i.e., $(A+B F) \xi \in \mathcal{X}_{f}$ for all $\xi \in \mathcal{X}_{f}$.

Assumption III.2 (Terminal set): For all $\zeta \in \mathcal{X}_{f}$, the following conditions hold:

$$
\zeta \in \mathcal{X}_{N-1} \cap \mathcal{T}_{N-1}^{\mathbb{X}} \quad F \zeta \in \mathcal{U}_{N-1} \cap \mathcal{T}_{N-1}^{\mathbb{U}}
$$

For the sake of notational simplicity, let us define $\mathscr{U}_{N}:=$ $\prod_{i=0}^{N-1} \mathcal{U}_{i}$ and $\mathscr{X}_{N}:=\prod_{i=0}^{N-1} \mathcal{X}_{i} \times \mathcal{X}_{f}$. The cost function of the $\mathrm{RMPC}$ problem is

$$
\begin{aligned}
V_{N}(x, \boldsymbol{u}):= & \sum_{i=0}^{N-1} d_{Q}\left(\phi_{i}^{\boldsymbol{u}, \mathbf{0}}(x), \mathcal{T}_{i}^{\mathbb{X}}\right)+d_{R}\left(u_{i}, \mathcal{T}_{i}^{\mathbb{U}}\right) \\
& +\delta_{\text {feas }}\left(\boldsymbol{u}, \boldsymbol{\phi}^{\boldsymbol{u}, \mathbf{0}}(x)\right)
\end{aligned}
$$

where $\delta_{\text {feas }}\left(\boldsymbol{u}, \boldsymbol{\phi}^{\boldsymbol{u}, \mathbf{0}}(x)\right)=0$ if $\boldsymbol{u} \in \mathscr{U}_{N}$ and $\boldsymbol{\phi}^{\boldsymbol{u}, \mathbf{0}}(x) \in \mathscr{X}_{N}$, and $=\infty$ otherwise, is the indicator function of the set $\mathscr{U}_{N} \times$ $\mathscr{X}_{N}$. The input and state constraints are embedded in the objective function via the indicator function. The optimization problem for a finite horizon $N$ with an initial state $x$ reads as

$$
V_{N}^{*}(x):=\min _{\boldsymbol{u}} V_{N}(x, \boldsymbol{u})
$$

with $\boldsymbol{u}^{\mathrm{mpc}}(x):=\operatorname{argmin}_{\boldsymbol{u}} V_{N}(x, \boldsymbol{u})$ as the optimal input sequence. When it is clear from the context, we may instead use the shorthand notation $\boldsymbol{u}^{\mathrm{mpc}}$. The above-mentioned sequence of inputs is indeed an optimal solution to a nominal (i.e., $\boldsymbol{w}=\mathbf{0}$ ) finite optimization problem emerging in the context of finite horizon MPC in the rest of this paper. In this light, we denote this nominally optimal controller by a similar label, for which the associated nominal state sequence is $\phi^{\mathbf{u}^{\mathrm{mpc}}, \mathbf{0}}(x)$.

In a standard RMPC setting, the optimal control problem (5) is solved. The first element $u_{0}^{\mathrm{mpc}}(x)$ of $\boldsymbol{u}^{\mathrm{mpc}}(x)$ is then applied to the plant yielding to the closed-loop dynamics $x^{+}=A x+$ $B u_{0}^{\mathrm{mpc}}(x)+w$. In an event-based setting, the triggering mechanism generally exploits the optimal state sequence $\boldsymbol{\phi}^{\boldsymbol{u}^{\mathrm{mpc}}, \mathbf{0}}(x)$ in order to possibly employ the rest of the elements in the nominally optimal input vector $\boldsymbol{u}^{\mathrm{mpc}}(x)$. The challenge in designing the triggering mechanism is then to guarantee robust stability and robust recursive feasibility of the resulting event-triggered, closed-loop dynamics.

Definition III.3 (Triggering mechanism): Given an initial state $x$ and a sequence of (possibly) state-dependent, hyperrectangular sets $\mathcal{E}(x):=\mathcal{E}_{0} \cup\left\{\mathcal{E}_{i}(x)\right\}_{i=1}^{N-1} \subset\left(\mathbb{R}^{n_{x}}\right)^{N}$, the triggering instance is defined by

$$
\begin{aligned}
k_{\text {trig }}^{\boldsymbol{w}}(x):=\min \{j \in\{0: N-1\}: & \\
& \left.\phi_{j}^{\boldsymbol{u}^{\mathrm{mpc}}, \boldsymbol{w}}(x)-\phi_{j}^{\boldsymbol{u}^{\mathrm{mpc}}, \mathbf{0}}(x) \notin \mathcal{E}_{j}(x)\right\}
\end{aligned}
$$

where $\mathcal{E}_{0}:=\mathbb{R}^{n_{x}}$.

The quantity $k_{\text {trig }}^{\boldsymbol{w}}(x)$ is known as the interexecution time in the literature. One can observe that $\phi_{0}^{\boldsymbol{u}^{\mathrm{mpc}}, \boldsymbol{w}}(x)=\phi_{0}^{\boldsymbol{u}^{\mathrm{mpc}}, \mathbf{0}}(x)=$ $x$. As a result, $\phi_{0}^{\boldsymbol{u}^{\mathrm{mpc}}, \boldsymbol{w}}(x)-\phi_{0}^{\boldsymbol{u}^{\mathrm{mpc}}, \mathbf{0}}(x)=0 \in \mathbb{R}^{n_{x}}=\mathcal{E}_{0}$, and thus, $k_{\text {trig }}^{\boldsymbol{w}}(x) \geq 1$. The closed-loop dynamics is then, for all $t \in$ $\mathbb{Z}_{\geq 0}$

$$
\begin{aligned}
& \xi_{t+1}=A \xi_{t}+B u_{t-\tau_{t}}^{\mathrm{mpc}}\left(\xi_{\tau_{t}}\right)+w_{t} \\
& \tau_{t+1}=\left\{\begin{array}{l}
\tau_{t},\left\{\begin{array}{l}
t-\tau_{t} \leq N-1 \& \\
\xi_{t}-\phi_{t-\tau_{t}}^{\boldsymbol{u}_{\mathrm{mpc}}}, \mathbf{o}
\end{array}\left(\xi_{\tau_{t}}\right) \in \mathcal{E}_{t-\tau_{t}}\left(\xi_{\tau_{t}}\right)\right. \\
t, \quad \text { otherwise }
\end{array}\right.
\end{aligned}
$$

given the initial state $\xi_{0}$ and the initial triggering instance $\tau_{0}=0$. Here, $\tau_{t}$ denotes the last triggering instance up to time $t$. Also, notice that a mandatory triggering is put in place at time $\tau_{t}+N$. The problem addressed in this paper is now introduced.

Problem III.4: Consider the closed-loop dynamics (7) under Assumptions III.1 and III.2. Devise an approach to construct the sequence of triggering sets $\mathcal{E}\left(\xi_{\tau_{t}}\right)$ in (6) such that the trajectories of the closed-loop dynamics satisfy:

1) Recursive feasibility: If $V_{N}^{*}\left(\xi_{0}\right)<\infty$, then $V_{N}^{*}\left(\xi_{t}\right)<\infty$, for all $t \in \mathbb{Z}_{>0}$;

2) Robust stability: The states and inputs of the closed-loop dynamics converge to the target sets $\mathbb{T}^{\mathbb{X}}$ and $\mathbb{T}^{\mathbb{U}}$, respectively, $\left(\lim _{t \rightarrow \infty} V_{N}^{*}\left(\xi_{t}\right)=0\right)$.

Remark III.5 (Smart actuators and sensors): The actuator and sensor units are "smart" in the following sense. The actuator (sensor) units can buffer the time-stamped and packetized sequence $\boldsymbol{u}^{\mathrm{mpc}}\left(\xi_{\tau_{t}}\right) \quad\left(\left\{\phi_{s}^{\boldsymbol{u}^{\mathrm{mpc}}, \mathbf{0}}\left(\xi_{\tau_{t}}\right) \oplus \mathcal{E}_{s}\left(\xi_{\tau_{t}}\right)\right\}_{s=1}^{N-1}\right)$. The actuator units consecutively apply the input action $u_{s-\tau_{t}}^{\mathrm{mpc}}\left(\xi_{\tau_{t}}\right)$ on the plant at each time $s \in\left\{\tau_{t}: \tau_{t+1}-1\right\}$. The sensor units evaluate the triggering condition

$$
\xi_{s} \notin \phi_{s-\tau_{t}}^{\boldsymbol{u}_{\mathrm{mpc}} \mathbf{0}}\left(\xi_{\tau_{t}}\right) \oplus \mathcal{E}_{s-\tau_{t}}\left(\xi_{\tau_{t}}\right)
$$

at each time $s \in\left\{\tau_{t}+1: \tau_{t}+N-1\right\}$. When the triggering condition holds at some time $s$, the sensors send the most recent 
states $\xi_{s}$ to the controller and the triggering instance is set to $\tau_{t+1}=s$.

Remark III.6 (Iteration complexity): RMPC problems with linear dynamics, a quadratic cost function, and polytopic constraints are quadratic programs for which dedicated solvers provide the complexity per iteration $\mathcal{O}\left(N\left(n_{x}+n_{u}\right)^{3}\right)$ [34].

\section{Main Results}

In this section, we provide several approaches to construct the sequence of sets $\mathcal{E}(x)$ which meets the requirements of Problem III.4. To this end, we begin by describing a certain type of constrained optimization problem that produces $\mathcal{E}(x)$. Based on these constructed sets, we then state the main theoretical results of this paper.

\section{A. Construction of Hyperrectangles $\mathcal{E}(x)$}

Let $j \in\{1: N-1\}$. The procedure to construct each hyperrectangle $\mathcal{E}_{j}(x)$ comprises the parametric representation of $\mathcal{E}_{j}(x)$, the definition of auxiliary quantities associated with $\mathcal{E}_{j}(x)$, and finally, the optimization problem to find $\mathcal{E}_{j}(x)$.

Notice that one way to represent a hyperrectangle $\mathcal{E}_{j}(x)$ is

$$
\mathcal{E}_{j}(x):=\left\{\epsilon \in \mathbb{R}^{n_{x}}:-\underline{e}_{j}(x) \leq \epsilon \leq \bar{e}_{j}(x)\right\}
$$

for some vectors $\underline{e}_{j}(x), \bar{e}_{j}(x) \in \mathbb{R}_{\geq 0}^{n_{x}}$. In other words, each hyperrectangle $\mathcal{E}_{j}(x)$ is parameterized by $2 n_{x}$ entries of $\underline{e}_{j}(x)$ and $\bar{e}_{j}(x)$.

Let us now introduce the auxiliary quantities involved in the derivation of $\mathcal{E}_{j}(x)$. Let $A_{\mathrm{cl}}:=(A+B F)$ be the nominal, closed-loop state matrix. Define the input sequence $\tilde{\boldsymbol{u}}(x ; j)$ and the associated state sequence $\phi^{\tilde{\boldsymbol{u}}, \mathbf{0}}(x ; j)$ as

$$
\begin{array}{r}
\tilde{u}_{i}(x ; j):=\left\{\begin{array}{lr}
u_{j+i}^{\mathrm{mpc}}(x), & i \in\{0: N-j-1\} \\
F A_{\mathrm{cl}}^{j+i-N} \phi_{N}^{\boldsymbol{u}^{\mathrm{mpc}}, \mathbf{0}}(x), & i \in\{N-j: N-1\}
\end{array}\right. \\
\phi_{i}^{\tilde{\boldsymbol{u}}, \mathbf{0}}(x ; j):=\left\{\begin{array}{lr}
\phi_{j+i}^{\boldsymbol{u}^{\mathrm{mpc}}, \mathbf{0}}(x), & i \in\{0: N-j\} \\
A_{\mathrm{cl}}^{j+i-N} \phi_{N}^{\boldsymbol{u}^{\mathrm{mpc}}, \mathbf{0}}(x), & i \in\{N-j+1: N\} .
\end{array}\right.
\end{array}
$$

The above-mentioned candidate input sequence is constructed by concatenating the last $N-j$ elements of $\boldsymbol{u}^{\mathrm{mpc}}(x)$ with the nominal feedback $F$ (recursively) applied to the optimal terminal state $\phi_{N}^{\boldsymbol{u}^{\mathrm{mpc}}, \mathbf{0}}(x)$.

Define $\mathscr{T}_{N}^{\mathbb{U}}:=\prod_{i=0}^{N-1} \mathcal{T}_{i}^{\mathbb{U}}$ and $\mathscr{T}_{N}^{\mathbb{X}}:=\prod_{i=0}^{N-1} \mathcal{T}_{i}^{\mathbb{X}}$. Denote now the projections of optimal state and input sequences $\boldsymbol{\phi}^{\boldsymbol{u}^{\mathrm{mpc}}, \mathbf{0}}(x)$ and $\boldsymbol{u}^{\mathrm{mpc}}(x)$ onto their corresponding target sets by $s^{\mathbb{X}}(x) \in \mathscr{T}_{N}^{\mathbb{X}}$ and $s^{\mathbb{U}}(x) \in \mathscr{T}_{N}^{\mathbb{U}}$, where for all $i \in\{0: N-1\}$

$s_{i}^{\mathbb{X}}(x):=\Pi_{Q}\left(\phi_{i}^{\boldsymbol{u}^{\mathrm{mpc}}, \mathbf{0}}(x), \mathcal{T}_{i}^{\mathbb{X}}\right) \quad s_{i}^{\mathbb{U}}(x):=\Pi_{R}\left(u_{i}^{\mathrm{mpc}}(x), \mathcal{T}_{i}^{\mathbb{U}}\right)$.

Based on the above definition, the next two auxiliary quantities are defined as follows. Let $\tilde{\boldsymbol{s}}^{\mathbb{U}}(x ; j)$ and $\tilde{\boldsymbol{s}}^{\mathbb{X}}(x ; j)$ represent the projection of $\tilde{\boldsymbol{u}}(x ; j)$ and $\phi^{\tilde{\boldsymbol{u}}, \mathbf{0}}(x ; j)$ onto $\mathscr{T}_{N}^{\mathbb{U}}$ and $\mathscr{T}_{N}^{\mathbb{X}}$, respectively. We have

$$
\begin{aligned}
& \tilde{s}_{i}^{\mathbb{U}}(x ; j):=\left\{\begin{array}{lc}
s_{j+i}^{\mathbb{U}}(x), & i \in\{0: N-j-1\} \\
\tilde{u}_{i+j}(x ; j), & i \in\{N-j: N-1\}
\end{array}\right. \\
& \tilde{s}_{i}^{\mathbb{X}}(x ; j):=\left\{\begin{array}{lr}
s_{j+i}^{\mathbb{X}}(x), & i \in\{0: N-j\} \\
\phi_{j+i}^{\tilde{\boldsymbol{u}}, \mathbf{0}}(x ; j), & i \in\{N-j+1: N-1\} .
\end{array}\right.
\end{aligned}
$$

Let us clarify the conventions used in (9). The definition of $\tilde{u}_{i}(x ; j)$ in (8a) implies that $\tilde{u}_{i}(x ; j) \in \mathcal{T}_{N-1}^{\mathbb{U}} \subseteq \mathcal{T}_{i}^{\mathbb{U}}$, for all $i \in$ $\{N-j: N-1\}$. That is, the distance $d_{R}\left(\tilde{u}_{i}(x ; j), \mathcal{T}_{i}^{\mathbb{U}}\right)=0$, and hence, $\Pi_{R}\left(\tilde{u}_{i}(x ; j), \mathcal{T}_{i}^{\mathbb{U}}\right)=\tilde{u}_{i}(x ; j)$, as given in (9a). A similar line of reasoning has been used in $(9 \mathrm{~b})$.

We next adopt the feedback gains $\tilde{K}_{i}$ and the state-transition matrices $\tilde{L}_{i}$ defined as

$$
\begin{aligned}
& \tilde{K}_{0}=0_{n_{u} \times n_{x}}, \tilde{K}_{i+1}=K_{i}, \forall i \in\{0: N-2\} \\
& \tilde{L}_{0}=\mathrm{I}_{n_{x}}, \tilde{L}_{i+1}=\left(A+B \tilde{K}_{i}\right) \tilde{L}_{i}, \forall i \in\{0: N-1\} .
\end{aligned}
$$

In the following, we use the matrices (10) to identify certain sets around the optimal state sequence $\phi^{\boldsymbol{u}^{\mathrm{mpc}}, \mathbf{0}}(x)$. These sets in turn will be used to formulate recursive feasibility and robust stability for the event-triggering setting (see the problem (12) and Section V-A).

Let us now provide two definitions for the volume of $\mathcal{E}_{j}(x)$, that are

$$
\begin{aligned}
& \operatorname{vol}_{1}\left(\mathcal{E}_{j}(x)\right):=\prod_{p \in\left\{1: n_{x}\right\}}\left(\bar{e}_{j}^{p}(x)+\underline{e}_{j}^{p}(x)\right) \\
& \operatorname{vol}_{2}\left(\mathcal{E}_{j}(x)\right):=\prod_{p \in\left\{1: n_{x}\right\}}\left(\bar{e}_{j}^{p}(x) \times \underline{e}_{j}^{p}(x)\right)
\end{aligned}
$$

where $\bar{e}_{j}^{p}(x)$ (resp. $\underline{e}_{j}^{p}(x)$ ) denotes the $p$ th entry of $\bar{e}_{j}(x)$ (resp. $\underline{e}_{j}(x)$ ). Notice that (11a) is the standard definition of volume for $\mathcal{E}_{j}(x)$ in $\mathbb{R}^{n_{x}}$. As it will be discussed later on, the application of (11a) to construct $\mathcal{E}_{j}(x)$ leads to a more asymmetric spread of $\mathcal{E}_{j}(x)$ around $\phi_{j}^{\boldsymbol{u}^{\mathrm{mpc}}, \mathbf{0}}(x)$ compared to the application of (11b). The asymmetry in turn implies that the triggering mechanism has no robustness in certain error directions, see Remark IV.7 for further details. Nonetheless, the definition (11a) leads to the construction of sets that have the maximum possible volume, in particular, higher than the ones constructed based on (11b).

For all $j \in\{1: N-1\}$, the problem to find each $\mathcal{E}_{j}(x)$ is

$$
\max _{\bar{e}_{j}(x), \underline{e}_{j}(x) \geq 0} \operatorname{vol}_{q}\left(\mathcal{E}_{j}(x)\right)
$$

s.t.

$$
\begin{array}{ll}
\phi_{i}^{\tilde{\boldsymbol{u}}, \mathbf{0}}(x ; j) \in \mathcal{X}_{i} \ominus \tilde{L}_{i} \mathcal{E}_{j}(x), & \forall i \in\{0: N-1\} \\
\tilde{u}_{i}(x ; j) \in \mathcal{U}_{i} \ominus \tilde{K}_{i} \tilde{L}_{i} \mathcal{E}_{j}(x), & \forall i \in\{0: N-1\} \\
\tilde{s}_{i}^{\mathbb{X}}(x ; j) \in \mathcal{T}_{i}^{\mathbb{X}} \ominus \tilde{L}_{i} \mathcal{E}_{j}(x), & \forall i \in\{0: N-1\} \\
\tilde{s}_{i}^{\mathbb{U}}(x ; j) \in \mathcal{T}_{i}^{\mathbb{U}} \ominus \tilde{K}_{i} \tilde{L}_{i} \mathcal{E}_{j}(x), & \forall i \in\{0: N-1\}
\end{array}
$$

where $q \in\{1,2\}$ determines which type of the volume definition in (11) is chosen. Notice that the objective function $\operatorname{vol}_{q}\left(\mathcal{E}_{j}(x)\right)$ is a nonlinear, nonconvex function with a decision 
variable $\mathcal{E}_{j}(x)$. Hence, the problem (12) is difficult to solve. In the next subsection, we show that this problem remains practically solvable, in particular, the set-based constraints (12b)-(12e) are effectively representable by linear inequalities (i.e., polytopic inequalities) such that: 1) the optimization problem (12) has a CP counterpart (in Theorem IV.4) and 2) the optimization problem (12) admits an LP relaxation (in Theorem IV.5).

\section{B. Event-Based Implementation}

We first show that the robust stability of the event-triggered, closed-loop dynamics (7) is guaranteed, which in turn leads to recursive feasibility of the closed-loop system. The triggering mechanism (6) is constructed by the approach proposed in (12). We next establish that the nonconvex problem (12) to construct the hyperrectangles $\mathcal{E}(x)$, has a $\mathrm{CP}$ reformulation and an LP relaxation, and therefore, can be efficiently solved in practice.

Theorem IV.1 (Robust convergence): Consider the closedloop dynamics (7), and suppose that the initial state $\xi_{0}$ is feasible (i.e., $\left.V_{N}^{*}\left(\xi_{0}\right)<\infty\right)$ ). For all $s \in\left\{\tau_{t}+1: \tau_{t+1}\right\}$, there exists an input sequence $\boldsymbol{u} \in \mathscr{U}_{N}$ such that

$$
\begin{aligned}
& V_{N}^{*}\left(\xi_{\tau_{t+1}}\right)-V_{N}^{*}\left(\xi_{\tau_{t}}\right) \leq V_{N}\left(\xi_{s}, \boldsymbol{u}\left(\xi_{s}\right)\right)-V_{N}^{*}\left(\xi_{\tau_{t}}\right) \\
& \leq-\left(\sum_{k=0}^{s-\tau_{t}-1} d_{Q}\left(\phi_{k}^{\boldsymbol{u}^{\mathrm{mpc}}, \mathbf{0}}\left(\xi_{\tau_{t}}\right), \mathcal{T}_{k}^{\mathbb{X}}\right)+d_{R}\left(u_{k}^{\mathrm{mpc}}\left(\xi_{\tau_{t}}\right), \mathcal{T}_{k}^{\mathbb{U}}\right)\right)
\end{aligned}
$$

In particular, the closed-loop dynamics (7) is asymptotically stable, i.e., $\lim _{t \rightarrow \infty} V_{N}^{*}\left(\xi_{t}\right)=0$.

Remark IV.2 (Recursive feasibility): The second inequality in (13) implies that $V_{N}\left(\xi_{s}, \boldsymbol{u}\left(\xi_{s}\right)\right)<\infty$, for all $s \in\left\{\tau_{t}+1\right.$ : $\left.\tau_{t+1}\right\}$. In other words, the optimization problem (5) remains feasible for all time $t \in \mathbb{Z}_{>0}$.

Remark IV.3 (Transmission protocol): We assume that all sensor and actuator units are clock synchronized. When the problem (5) is solved, the controller node sends: 1) $\boldsymbol{u}^{\mathrm{mpc}}\left(\xi_{\tau_{t}}\right)$ to the actuator nodes and 2) each entry of $\phi_{j}^{\boldsymbol{u}^{\mathrm{mpc}}, \mathbf{0}}\left(\xi_{\tau_{t}}\right)-\underline{e}_{j}\left(\xi_{\tau_{t}}\right)$ and $\phi_{j}^{\boldsymbol{u}^{\mathrm{mpc}}, \mathbf{0}}\left(\xi_{\tau_{t}}\right)+\bar{e}_{j}\left(\xi_{\tau_{t}}\right)$ to the corresponding sensory nodes, for all $j \in\{1: N-1\}$. Moreover, the $n_{x}$ sensor units declare a triggering instance to each other, through a cost-efficient shortrange transmission. Then, all sensors declare their time-stamped, observed states to the controller.

The successful usage of the above-mentioned results is conditioned upon the premise that there exist computationally tractable methods to construct the sets $\mathcal{E}(x)$. We now revisit problem (12) to show that such a premise is valid by providing two frameworks - one in a $\mathrm{CP}$ form and another one in an LP form. In these frameworks, the parametric-in-set constraints (12b)-(12e) can be reformulated into a new set of linear inequalities in terms of the vertices of each set $\mathcal{E}_{j}(x)$. We shall call the polytope represented by the derived linear inequalities, the principal polytope $\overline{\mathcal{S}}$. Both frameworks try to find a maximum-volume hyperrectangle $\mathcal{E}_{j}(x)$ inscribed (or contained) in the principal polytope such that $0 \in \mathcal{E}_{j}(x)$. In the LP framework, we partly employ some results from [37], see
Section V-B and avoid reiterating the proofs of borrowed material. For notational convenience, let $\xi \in \mathcal{S} \ominus M \mathcal{B}(l, u)$ represent a concatenated version of the constraint $(12 \mathrm{~b})-(12 \mathrm{e})$ where, in particular, $\mathcal{B}(l, u):=\mathcal{E}_{j}(x)$. Hereafter, when we take the volume (of a hyperrectangle) as defined in (11), index $q=1$ and $q=2$ refers to (11a) and (11b), respectively.

Theorem IV.4 (Volume maximization-CP reformulation): Consider a vector $\xi \in \mathbb{R}^{p}$, a matrix $M \in \mathbb{R}^{p \times k}$, and a polytope $\mathcal{S}=\left\{s \in \mathbb{R}^{p}: A_{\mathcal{S}} s \leq b_{\mathcal{S}}\right\}$ containing the origin where $A_{\mathcal{S}} \in \mathbb{R}^{m \times p}$ and $b_{\mathcal{S}} \in \mathbb{R}^{m}$. The maximum volume hyperrectangle $\mathcal{B}(l, u) \subset \mathbb{R}^{k}$ that contains the origin and satisfies $\xi \in \mathcal{S} \ominus M \mathcal{B}(l, u)$ is $\mathcal{B}\left(-\underline{v}^{*}, \bar{v}^{*}\right)$ where $\underline{v}^{*}$ and $\bar{v}^{*}$ are the optimal solutions of the problem

$$
\begin{array}{ll}
\min _{\underline{v}, \bar{v}} & f_{q}(\bar{v}, \underline{v}) \\
\text { s.t. } & \left\langle w^{i},\left[\bar{v}^{\top} \underline{v}^{\top}\right]^{\top}\right\rangle \leq b_{i, \mathcal{S}}-a_{i, \mathcal{S}} \xi, \forall i \in\{1: m\} \\
& \bar{v} \geq 0, \underline{v} \geq 0
\end{array}
$$

where for $q \in\{1,2\}$

$$
\begin{aligned}
& f_{1}(\bar{v}, \underline{v}):=-\sum_{j \in\{1: k\}} \log \left(\bar{v}_{j}+\underline{v}_{j}\right) \\
& f_{2}(\bar{v}, \underline{v}):=-\sum_{j \in\{1: k\}} \log \left(\bar{v}_{j}\right)+\log \left(\underline{v}_{j}\right)
\end{aligned}
$$

and for all $j \in\{1: k\}$

$$
\begin{aligned}
w_{j}^{i} & = \begin{cases}\left(M^{\top} a_{i, \mathcal{S}}^{\top}\right)_{j}, & \text { if } \hat{w}_{j}^{i}=1 \\
0, & \text { otherwise }\end{cases} \\
w_{k+j}^{i} & = \begin{cases}-\left(M^{\top} a_{i, \mathcal{S}}^{\top}\right)_{j}, & \text { if } \hat{w}_{j}^{i}=-1 \\
0, & \text { otherwise }\end{cases}
\end{aligned}
$$

with $\hat{w}^{i}:=\operatorname{sign}\left(M^{\top} a_{i, \mathcal{S}}^{\top}\right)$, for all $i \in\{1: m\}$.

Theorem IV.5 (Volume maximization $-L P$ relaxation):

Suppose the hypotheses in Theorem IV.4 hold.

1) $(\mathbf{q}=\mathbf{1})$ The maximum volume $r$-constrained hyperrectangle $\mathcal{B}(l, u) \subset \mathbb{R}^{k}$ that contains the origin and satisfies $\xi \in \mathcal{S} \ominus M \mathcal{B}(l, u)$ is $\mathcal{B}\left(z^{*}, z^{*}+\lambda^{*} r\right)$ for which $z^{*} \in \mathbb{R}^{k}$ and $\lambda^{*} \in \mathbb{R}$ are the optimal solutions of

$$
\begin{aligned}
\max _{z, \lambda} & \lambda \\
\text { s.t. } & A_{\mathcal{S}} M z+\left(A_{\mathcal{S}} M\right)^{+} r \lambda \leq b_{\mathcal{S}}-A_{\mathcal{S}} \xi \\
& z+\lambda r \geq 0, z \leq 0
\end{aligned}
$$

where the $j$ th entry of $r, j \in\{1: k\}$, is defined as

$$
\begin{aligned}
r_{j}(\overline{\mathcal{S}}):=\max _{z, \omega} & \omega \\
\text { s.t. } & A_{\mathcal{S}} M z \leq b_{\mathcal{S}}-A_{\mathcal{S}} \xi \\
& A_{\mathcal{S}} M\left(z+\omega e_{j}\right) \leq b_{\mathcal{S}}-A_{\mathcal{S}} \xi \\
& z+\omega e_{j} \geq 0, z \leq 0
\end{aligned}
$$

where $e_{j} \in \mathbb{R}^{k}$ is the unit vector in the $j$ th direction and the polytope $\overline{\mathcal{S}}$ is

$$
\overline{\mathcal{S}}:=\left\{z \in \mathbb{R}^{k}: A_{\mathcal{S}} M z \leq b_{\mathcal{S}}-A_{\mathcal{S}} \xi\right\} .
$$


2) $(\mathbf{q}=\mathbf{2})$ The maximum volume $r$-constrained hyperrectangle $\mathcal{B}(l, u) \subset \mathbb{R}^{k}$ that contains the origin and satisfies $\xi \in \mathcal{S} \ominus M \mathcal{B}(l, u)$ is $\mathcal{B}\left(-\lambda^{*} r_{1}, \lambda^{*} r_{2}\right)$ for which $\lambda^{*} \in \mathbb{R}$ is the optimal solution of

$$
\begin{array}{ll}
\max _{\lambda} & \lambda \\
\text { s.t. } & (W)^{+} r \lambda \leq B
\end{array}
$$

where $r=\left(r_{2}^{\top}, r_{1}^{\top}\right)^{\top}$ and the $j$ th entry of $r, j \in\{1: 2 k\}$, is defined as

$$
\begin{aligned}
r_{j}:=\max _{\omega} & \omega \\
\text { s.t. } & W^{\prime}\left(\omega e_{j}\right) \leq B^{\prime}
\end{aligned}
$$

where $e_{j} \in \mathbb{R}^{2 k}$ is the unit vector in the $j$ th direction

$$
\begin{array}{lr}
W=\left(w^{1}, \ldots, w^{m}\right)^{\top} & W^{\prime}=\left(\begin{array}{cc}
W & \\
-\mathrm{I}_{k} & 0_{k \times 1} \\
0_{k \times 1} & -\mathrm{I}_{k}
\end{array}\right) \\
B=b_{\mathcal{S}}-A_{\mathcal{S}} \xi & B^{\prime}=\left(B^{\top}, 0_{1} \times 2 k\right)^{\top}
\end{array}
$$

and for all $i \in\{1: m\}, w^{i}$ are defined in (16).

We should emphasize that although Theorems IV.4 and IV.5 provide a way to construct $\mathcal{E}_{j}(x)$ with a maximal volume, the derived set is not unique (the corresponding cost functions of these approaches are not strictly convex to guarantee the uniqueness of the solution). In the remainder of the paper, we denote the construction approach based on the CP (14) with $q=1$ and $q=2$ by $\mathrm{CP}_{1}$ and $\mathrm{CP}_{2}$, respectively. Furthermore, $\mathrm{LP}_{1}$ represents the $\mathrm{LP}$ relaxation (17) of $\mathrm{CP}_{1}$ and $\mathrm{LP}_{2}$ denotes the $\mathrm{LP}$ relaxation (18) of $\mathrm{CP}_{2}$.

\section{Further Comments on Complexity and Sensitivity}

In the rest of this section, we allude briefly to two important practical aspects of the proposed construction approaches and possible directions to improve them. First, since these approaches are implemented online, they require an extra computation step besides the computation of the optimal input sequence. Fixed-thresholding approaches in the literature, for example [25], avoid this extra step by considering predefined triggering sets. We provide the arithmetic complexity of the proposed approaches to quantify the extra computational burden. To this end, we adopt the following notion of an oracle to represent the optimization problems in this paper. Let $A \in \mathbb{R}^{n_{c} \times n_{d}}$, $b \in \mathbb{R}^{n_{c}}, c \in \mathbb{R}^{n_{d}}$, and $f: \mathbb{R}^{n_{d}} \rightarrow \mathbb{R}$ be a concave function. Also, let $\mathbf{l p}\left(n_{c}, n_{d}\right)$ denote the oracle complexity for solving $\max _{\eta}\left\{c^{\top} \eta: A \eta \leq b\right\}$, and $\operatorname{cp}\left(n_{c}, n_{d}\right)$ denote the oracle complexity for solving $\max _{\eta}\{f(\eta): A \eta \leq b\}$.

Remark IV.6 (Computational complexity): The oracle complexity of the CP reformulations (14) in Theorem IV.4 is $\mathbf{c p}(m+$ $2 k, 2 k)$ and of the LP reformulations (17) and (18) in Theorem IV.5 are $\operatorname{lp}(m+2 k, k+1)+k \times \operatorname{lp}(2 m+2 k, k+1)$ and $\mathbf{l} \mathbf{p}(m, 1)+2 k \times \operatorname{lp}(m+2 k, 1)$, respectively. A possible remedy to circumvent these computations is to introduce a

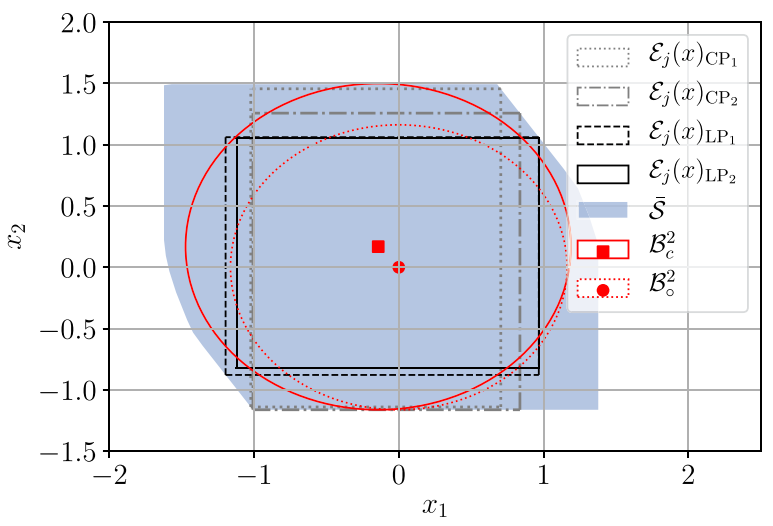

(a)

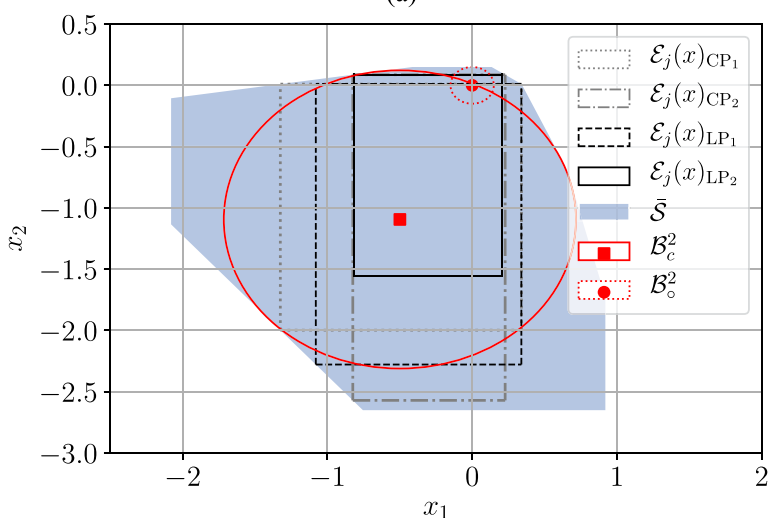

(b)

Fig. 1. Comparison of the CP and LP approaches to construct $\mathcal{E}_{j}(x) \subseteq$ $\overline{\mathcal{S}}$. (a) $\overline{\mathcal{S}}$ is distributed in a fairly uniform manner around the origin. All the approaches provide close behaviors. (b) $\overline{\mathcal{S}}$ is distributed in a relatively uneven manner around the origin. The approaches $\mathrm{CP}_{2}$ and $\mathrm{LP}_{2}$ promote more symmetric constructions compared to the approaches $\mathrm{CP}_{1}$ and $\mathrm{LP}_{1}$. (a) Well-shaped polytope example, $\frac{r_{c}}{r_{0}}=1.0861$. (b) III-shaped polytope example, $\frac{r_{\mathrm{C}}}{r_{\mathrm{o}}}=8.0669$.

state-independent triggering law, as opposed to the current statedependent law (6). This extension would allow to compute the desired sets offline and only once.

The other issue regarding the proposed approaches is the asymmetry of the triggering sets with respect to the optimal state sequence. Let polytope $\overline{\mathcal{S}} \subset \mathbb{R}^{n_{x}}$ represent the constraints $(12 \mathrm{~b})-(12 \mathrm{e})$ that the triggering set $\mathcal{E}_{j}(x)$ satisfies. In other words, $\mathcal{E}_{j}(x)$ is constructed inside $\overline{\mathcal{S}}$. Recall that $\mathcal{E}_{j}(x)$ represents the "allowable" prediction error so that the triggering mechanism is not activated. Qualitatively speaking, for a "better" directional resilience against prediction errors, one would prefer symmetry in the constructed $\mathcal{E}_{j}(x)$. The above statements are schematically depicted in Fig. 1 . When $\overline{\mathcal{S}}$ is well shaped as in Fig. 1(a), the approaches in Theorems IV.4 and IV.5 lead to a relatively symmetric set $\mathcal{E}_{j}(x)$ with respect to the origin. When $\overline{\mathcal{S}}$ is ill shaped as in Fig. 1(b), the constructed set $\mathcal{E}_{j}(x)$ is, however, extremely asymmetric with respect to the origin along some coordinates. This difference is well captured by the geometric measure $\frac{r_{\mathrm{c}}}{r_{\mathrm{o}}}$ of $\overline{\mathcal{S}}$, where $r_{\mathrm{c}}$ is the radius of the maximal 2 -norm ball inside $\overline{\mathcal{S}}$, and $r_{\circ}$ is the radius of the maximal 2-norm ball, centered at the origin and inside $\overline{\mathcal{S}}$. By definition, we have 
$\frac{r_{\mathrm{c}}}{r_{\mathrm{O}}} \geq 1$. Observe that in well shaped cases $r_{c} / r_{\circ} \approx 1$ and in ill-shaped cases $r_{c} / r_{\circ} \gg 1$.

Remark IV.7 (Directional sensitivity to prediction errors): The directional sensitivity issue is the main reason for introducing the second definition (11b) of the volume. To see this, assume first that our goal is to maximize the log value of the volume of $\mathcal{E}_{j}(x)$. The first definition (11a) solely aims at maximizing the width of $\mathcal{E}_{j}(x)$ within $\overline{\mathcal{S}}$ along each coordinate. On the other hand, the second definition (11b) maximizes the width of $\mathcal{E}_{j}(x)$ in both positive and negative directions along each coordinate. As shown in Fig. 1, in both cases the set $\mathcal{E}_{j}(x)$ constructed by the approaches $\mathrm{CP}_{2}$ and $\mathrm{LP}_{2}$ is typically more symmetric compared to those constructed by the approaches $\mathrm{CP}_{1}$ and $\mathrm{LP}_{1}$. An interesting research direction to alleviate this sensitivity issue is to investigate the impact of the MPC design parameters (e.g., the tightening gains $\boldsymbol{K}$ or the target sets $\mathbb{T}^{\mathbb{X}}$ and $\mathbb{T}^{\mathbb{U}}$ ).

\section{TECHNICAL PROOFS}

\section{A. Proof of Theorem IV.1}

The proof consists of five main steps. Each step is labeled by the guaranteed property. Let $x:=\xi_{\tau_{t}}$ be the state at the last triggering instance. Define the prediction error

$$
e_{j}^{\boldsymbol{w}}(x)=\phi_{j}^{\boldsymbol{u}^{\mathrm{mpc}}, \boldsymbol{w}}(x)-\phi_{j}^{\boldsymbol{u}^{\mathrm{mpc}}, \mathbf{0}}(x)
$$

indicating the mismatch between the perturbed system and the nominal one. For some integer $j \in\{0: N-1\}$, suppose that the mechanism is enabled at time $j+1$, that is, either (1) $j<$ $N-1$ so that for all $i \in\{0: j\}, e_{i}^{\boldsymbol{w}}(x) \in \mathcal{E}_{i}(x)$ and $e_{j+1}^{\boldsymbol{w}}(x) \notin$ $\mathcal{E}_{j+1}(x)$, or (2) $j=N-1$ [see equation (7b)]. We omit the arguments of variables for convenience when it is clear from the context (unless mentioned otherwise). In what follows, we also use the notation $\ell\left(x_{i}, u_{i}\right)$ for $d_{Q}\left(x_{i}, \mathcal{T}_{i}^{\mathbb{X}}\right)+d_{R}\left(u_{i}, \mathcal{T}_{i}^{\mathbb{U}}\right)$ for notational simplicity.

1) Interevent recursive feasibility: Define the candidate input sequence $\boldsymbol{u}^{\mathrm{c}}(x ; j)$ such that for all $i \in\{0: N-1\}$

$$
u_{i}^{\mathrm{c}}:=\tilde{u}_{i}+\tilde{K}_{i} \tilde{L}_{i} e_{j}^{\boldsymbol{w}}
$$

and its associated candidate state sequence $\boldsymbol{\phi}^{\boldsymbol{u}^{\mathrm{c}}, \mathbf{0}}(x ; j)$, where for all $i \in\{0: N\}$

$$
\phi_{i}^{\boldsymbol{u}^{\mathrm{c}}, \mathbf{0}}:=\phi_{i}^{\tilde{\boldsymbol{u}}, \mathbf{0}}+\tilde{L}_{i} e_{j}^{\boldsymbol{w}} .
$$

Note that $\phi_{0}^{\boldsymbol{u}^{\mathrm{c}}, \mathbf{0}}=\phi_{j}^{\boldsymbol{u}^{\mathrm{mpc}}, \boldsymbol{w}}$ and $u_{0}^{\mathrm{c}}=u_{j}^{\mathrm{mpc}}$. We now establish that the sequences $\boldsymbol{u}^{\mathrm{c}}$ and $\phi^{\boldsymbol{u}^{\mathrm{c}}, \mathbf{0}}$ satisfy $\boldsymbol{u}^{\mathrm{c}} \in \mathscr{U}_{N}$ and $\boldsymbol{\phi}^{\boldsymbol{u}^{\mathrm{c}}, \boldsymbol{0}} \in \mathscr{X}_{N}$, i.e., $V_{N}\left(\phi_{j}^{\boldsymbol{u}^{\mathrm{mpc}}, \boldsymbol{w}}, \boldsymbol{u}^{\mathrm{c}}\right)<\infty$. By assumption, $e_{j}^{\boldsymbol{w}} \in \mathcal{E}_{j}$. Moreover, $\mathcal{E}_{j}$ satisfies (12b)-(12c). From the definition of the Pontryagin difference, it follows that $u_{i}^{\mathrm{c}} \in \mathcal{U}_{i}$ and $\phi_{i}^{\boldsymbol{u}^{\mathrm{c}}, \mathbf{0}} \in \mathcal{X}_{i}$, for all $i \in\{0: N-1\}$. Recall that $L_{N-1}=0$. Hence, $\tilde{L}_{N}=0$ and $\phi_{N}^{\boldsymbol{u}^{\mathrm{c}}, \mathbf{0}}=\phi_{N}^{\tilde{\boldsymbol{u}}, \mathbf{0}}$. From (8b), we have $\phi_{N}^{\tilde{\boldsymbol{u}}, \mathbf{0}}=A_{\mathrm{cl}}^{j} \phi_{N}^{\boldsymbol{u}^{\mathrm{mpc}}, \mathbf{0}}(x)$. Since $\mathcal{X}_{f}$ is a control invariant set, $\phi_{N}^{\boldsymbol{u}^{\mathrm{c}}, \mathbf{0}} \in \mathcal{X}_{f}$. We conclude that $V_{N}\left(\phi_{j}^{\boldsymbol{u}^{\mathrm{mpc}}, \boldsymbol{w}}, \boldsymbol{u}^{\mathrm{c}}\right)<\infty$.

2) Interevent cost function decay: Observe that

$$
\begin{aligned}
d_{Q}\left(\phi_{i}^{\boldsymbol{u}^{\mathrm{c}}, \mathbf{0}}, \mathcal{T}_{i}^{\mathbb{X}}\right) & =d_{Q}\left(\phi_{i}^{\tilde{\boldsymbol{u}}, \mathbf{0}}+\tilde{L}_{i} e_{j}^{\boldsymbol{w}}, \mathcal{T}_{i}^{\mathbb{X}}\right) \\
& \leq d_{Q}\left(\phi_{i}^{\tilde{\boldsymbol{u}}, \mathbf{0}}, \mathcal{T}_{i}^{\mathbb{X}} \ominus \tilde{L}_{i} \mathcal{E}_{j}\right)
\end{aligned}
$$

where we made use of the definition (20b) and Lemma II.1, respectively. Recall from (12d) that $\tilde{s}_{i}^{\mathbb{X}} \in \mathcal{T}_{i}^{\mathbb{X}} \ominus \tilde{L}_{i} \mathcal{E}_{j}$. Hence

$$
0 \leq d_{Q}\left(\phi_{i}^{\boldsymbol{u}^{\mathrm{c}}, \mathbf{0}}, \mathcal{T}_{i}^{\mathbb{X}}\right) \leq d_{Q}\left(\phi_{i}^{\tilde{\boldsymbol{u}}, \mathbf{0}}, \tilde{s}_{i}^{\mathbb{X}}\right) .
$$

Similarly, one can arrive at

$$
0 \leq d_{R}\left(u_{i}^{\mathrm{c}}, \mathcal{T}_{i}^{\mathbb{U}}\right) \leq d_{R}\left(\tilde{u}_{i}, \tilde{s}_{i}^{\mathbb{U}}\right) .
$$

Consider $i \in\{0: N-j-1\}$. In light of the definitions (8b) and (9b), we have $d_{Q}\left(\phi_{i}^{\tilde{\boldsymbol{u}}, \mathbf{0}}, \tilde{s}_{i}^{\mathbb{X}}\right)=d_{Q}\left(\phi_{j+i}^{\boldsymbol{u}^{\mathrm{mpc}}, \mathbf{0}}, \mathcal{T}_{j+i}^{\mathbb{X}}\right)$. Thus

$$
d_{Q}\left(\phi_{i}^{\boldsymbol{u}^{\mathrm{c}}, \mathbf{0}}, \mathcal{T}_{i}^{\mathbb{X}}\right) \leq d_{Q}\left(\phi_{j+i}^{\boldsymbol{u}^{\mathrm{mpc}}, \boldsymbol{w}}, \mathcal{T}_{j+i}^{\mathbb{X}}\right) .
$$

In a similar fashion, we can show

$$
d_{R}\left(u_{i}^{\mathrm{c}}, \mathcal{T}_{i}^{\mathbb{U}}\right) \leq d_{Q}\left(u_{j+i}^{\mathrm{mpc}}, \mathcal{T}_{j+i}^{\mathbb{U}}\right) .
$$

From (22), it is then straightforward that

$$
\sum_{i=0}^{N-j-1} \ell\left(\phi_{i}^{\boldsymbol{u}^{\mathrm{c}}, \mathbf{0}}, u_{i}^{\mathrm{c}}\right) \leq \sum_{i=0}^{N-j-1} \ell\left(\phi_{j+i}^{\boldsymbol{u}^{\mathrm{mpc}}, \mathbf{0}}, u_{j+i}^{\mathrm{mpc}}\right) .
$$

Now, let $i \in\{N-j: N-1\}$ and consider the definition (9). Then, $d_{Q}\left(\phi_{i}^{\tilde{\boldsymbol{u}}, \mathbf{0}}, \tilde{s}_{i}^{\mathbb{X}}\right)=d_{R}\left(\tilde{u}_{i}, \tilde{s}_{i}^{\mathbb{U}}\right)=0$. These equality relations coupled with (21) give rise to

$$
\sum_{i=N-j}^{N-1} \ell\left(\phi_{i}^{\boldsymbol{u}^{\mathrm{c}}, \mathbf{0}}, u_{i}^{\mathrm{c}}\right)=0 .
$$

From (23), we finally infer that if $e_{j}^{\boldsymbol{w}} \in \mathcal{E}_{j}$, then

$$
\begin{aligned}
& V_{N}\left(\phi_{j}^{\boldsymbol{u}^{\mathrm{mpc}}, \boldsymbol{w}}, \boldsymbol{u}^{\mathrm{c}}\right)=V_{N}\left(\phi_{0}^{\boldsymbol{u}^{\mathrm{c}}, \mathbf{0}}, \boldsymbol{u}^{\mathrm{c}}\right) \\
& \leq V_{N}^{*}(x)-\sum_{i=0}^{j-1} \ell\left(\phi_{i}^{\boldsymbol{u}^{\mathrm{mpc}}, \mathbf{0}}, u_{i}^{\mathrm{mpc}}\right) .
\end{aligned}
$$

3) At-event recursive feasibility: Consider now the new candidate input sequence $\hat{\boldsymbol{u}}^{\mathrm{c}}(x ; j+1)$ where

$$
\hat{u}_{i}^{\mathrm{c}}:=\left\{\begin{array}{lr}
u_{i+1}^{\mathrm{c}}+K_{i} L_{i} w_{j}, & \forall i \in\{0: N-2\} \\
F \phi_{N}^{\boldsymbol{u}^{\mathrm{c}}, \mathbf{0}}+F L_{N-1} w_{j}, & i=N-1
\end{array}\right.
$$

and its associated candidate state sequence $\hat{\boldsymbol{\phi}}^{\hat{\mathbf{u}}^{\mathrm{c}}, \mathbf{0}}(x ; j+1)$ such that

$$
\hat{\phi}_{i}^{\hat{\boldsymbol{u}}^{\mathrm{c}}, \mathbf{0}}:=\left\{\begin{array}{lr}
\phi_{i+1}^{\boldsymbol{u}^{\mathrm{c}}, \mathbf{0}}+L_{i} w_{j}, & \forall i \in\{0: N-1\} \\
A_{\mathrm{cl}} \hat{\phi}_{N-1}^{\hat{\boldsymbol{u}}^{\mathrm{c}}, \mathbf{0}}, & i=N
\end{array}\right.
$$

where $w_{j} \in \mathbb{W}$. Observe that

$$
\begin{aligned}
\hat{\phi}_{0}^{\hat{\boldsymbol{u}}^{\mathrm{c}}, \mathbf{0}} & =\phi_{1}^{\boldsymbol{u}^{\mathrm{c}}, \mathbf{0}}+w_{j}=A \phi_{0}^{\boldsymbol{u}^{\mathrm{c}}, \mathbf{0}}+B u_{0}^{\mathrm{c}}+w_{j} \\
& =A \phi_{j}^{\boldsymbol{u}^{\mathrm{mpc}}, \boldsymbol{w}}+B u_{j}^{\mathrm{mpc}}+w_{j}=\phi_{j+1}^{\boldsymbol{u}^{\mathrm{mpc}}, \boldsymbol{w}} .
\end{aligned}
$$

We now show that $\hat{\boldsymbol{u}}^{\mathrm{c}} \in \mathscr{U}_{N}$ and $\hat{\boldsymbol{\phi}}^{\hat{\boldsymbol{u}}^{\mathrm{c}}, \boldsymbol{0}} \in \mathscr{X}_{N}$, i.e., $V_{N}\left(\phi_{j+1}^{\boldsymbol{u}^{\mathrm{mpc}}, \boldsymbol{w}}, \hat{\boldsymbol{u}}^{\mathrm{c}}\right)<\infty$. Observe that $u_{i+1}^{\mathrm{c}} \in \mathcal{U}_{i+1}$ and $w_{j} \in \mathbb{W}$. Hence, $u_{i+1}^{\mathrm{c}} \in \mathcal{U}_{i+1} \oplus K_{i} L_{i} \mathbb{W}$ for all $i \in\{0: N-2\}$. Since $\mathcal{U}_{i+1}=\mathcal{U}_{i} \ominus K_{i} L_{i} \mathbb{W}$, we have $\hat{u}_{i}^{\mathrm{c}} \in \mathcal{U}_{i}$ for all $i \in\{0: N-2\}$. Recall now $\phi_{N}^{\boldsymbol{u}^{\mathrm{c}}, \mathbf{0}} \in \mathcal{X}_{f}$ (from Step 1). Assumption III.2 along with $L_{N-1}=0$ imply that $\hat{u}_{N-1}^{\mathrm{c}} \in \mathcal{U}_{N-1}$. We have $\phi_{i+1}^{\boldsymbol{u}^{\mathrm{c}}, \mathbf{0}} \in$ $\mathcal{X}_{i+1}$ for all $i \in\{0: N-2\}$. Then, $\hat{\phi}_{i}^{\hat{\boldsymbol{u}}^{\mathrm{c}}, \mathbf{0}} \in \mathcal{X}_{i+1} \oplus L_{i} \mathbb{W}$. For all $i \in\{0: N-2\}$, it follows from $\mathcal{X}_{i+1}=\mathcal{X}_{i} \ominus L_{i} \mathbb{W}$ that 
$\hat{\phi}_{i}^{\hat{\boldsymbol{u}}^{\mathrm{c}}, \mathbf{0}} \in \mathcal{X}_{i}$. Recall that $\phi_{N}^{\boldsymbol{u}^{\mathrm{c}}, \mathbf{0}} \in \mathcal{X}_{f}$ and $L_{N-1}=0$. Hence, we arrive at $\hat{\phi}_{N-1}^{\hat{\mathbf{u}}^{\mathrm{c}}, \mathbf{0}} \in \mathcal{X}_{f}$, and as a result, $\hat{\phi}_{N}^{\hat{\mathbf{u}}^{\mathrm{c}}, \mathbf{0}} \in \mathcal{X}_{f}$. We thus have $\hat{\boldsymbol{u}}^{\mathrm{c}} \in \mathscr{U}_{N}$ and $\hat{\boldsymbol{\phi}}^{\hat{\boldsymbol{u}}^{\mathrm{c}}, \mathbf{0}} \in \mathscr{X}_{N}$, i.e., $V_{N}\left(\phi_{j+1}^{\boldsymbol{u}^{\mathrm{mpc}}, \boldsymbol{w}}, \hat{\boldsymbol{u}}^{\mathrm{c}}\right)<\infty$.

4) At-event value function decay: Consider now $\hat{\boldsymbol{u}}^{\mathrm{c}}$ and $\hat{\boldsymbol{\phi}}^{\hat{\boldsymbol{u}}^{\mathrm{c}}, \mathbf{0}}$ as the candidate input and state sequences at time $j+1$, respectively. For all $i \in\{0: N-2\}$ and for all $w_{j} \in \mathbb{W}$

$$
\begin{aligned}
d_{Q}\left(\hat{\phi}_{i}^{\hat{\mathbf{u}}^{\mathrm{c}}, \mathbf{0}}, \mathcal{T}_{i}^{\mathbb{X}}\right) & =d_{Q}\left(\phi_{i+1}^{\boldsymbol{u}^{\mathrm{c}}, \mathbf{0}}+L_{i} w_{j}, \mathcal{T}_{i}^{\mathbb{X}}\right) \\
& \leq d_{Q}\left(\phi_{i+1}^{\boldsymbol{u}^{\mathrm{c}}, \mathbf{0}}, \mathcal{T}_{i}^{\mathbb{X}} \ominus \tilde{L}_{i} \mathcal{E}_{j}\right)=d_{Q}\left(\phi_{i+1}^{\boldsymbol{u}^{\mathrm{c}}, \mathbf{0}}, \mathcal{T}_{i+1}^{\mathbb{X}}\right)
\end{aligned}
$$

where the first inequality follows from (25b), the inequality is implied by Lemma II.1, and the second equality is derived from (3b). Following a similar argument, we arrive at

$$
d_{R}\left(\hat{u}_{i}^{\mathrm{c}}, \mathcal{T}_{i}^{\mathbb{U}}\right) \leq d_{R}\left(u_{i+1}^{\mathrm{c}}, \mathcal{T}_{i+1}^{\mathbb{U}}\right) .
$$

Since $L_{N-1}=0, \hat{\phi}_{N-1}^{\hat{\boldsymbol{u}}^{\mathrm{c}}, \mathbf{0}}=\phi_{N}^{\boldsymbol{u}^{\mathrm{c}}, \mathbf{0}}$ and $\hat{u}_{N-1}^{\mathrm{c}}=F \phi_{N}^{\boldsymbol{u}^{\mathrm{c}}, \mathbf{0}}$. In Step 3, it is shown that $\hat{\phi}_{N-1}^{\hat{\boldsymbol{u}}^{\mathrm{c}}, \mathbf{0}} \in \mathcal{X}_{f}$. Then, Assumption III.2 implies that $\hat{\phi}_{N-1}^{\hat{\boldsymbol{u}}^{\mathrm{c}}, \mathbf{0}} \in \mathcal{T}_{N-1}^{\mathbb{X}}$ and $\hat{u}_{N-1}^{\mathrm{c}} \in \mathcal{T}_{N-1}^{\mathbb{U}}$. Hence, $\ell\left(\hat{\phi}_{N-1}^{\hat{\boldsymbol{u}}^{\mathrm{c}}, \mathbf{0}}, \hat{u}_{N-1}^{\mathrm{c}}\right)=0$. By virtue of the inequalities in (26), we then arrive at

$$
\begin{aligned}
V_{N}\left(\phi_{j+1}^{\boldsymbol{u}^{\mathrm{mpc}}, \boldsymbol{w}}, \hat{\boldsymbol{u}}^{\mathrm{c}}\right) & =V_{N}\left(\hat{\phi}_{0}^{\hat{\boldsymbol{u}}^{\mathrm{c}}, \mathbf{0}}, \hat{\boldsymbol{u}}^{\mathrm{c}}\right) \leq \sum_{i=1}^{N-1} \ell\left(\phi_{i}^{\boldsymbol{u}^{\mathrm{c}}, \mathbf{0}}, u_{i}^{\mathrm{c}}\right) \\
& =V_{N}\left(\phi_{0}^{\boldsymbol{u}^{\mathrm{c}}, \mathbf{0}}, \boldsymbol{u}^{\mathrm{c}}\right)-\ell\left(\phi_{0}^{\boldsymbol{u}^{\mathrm{c}}, \mathbf{0}}, u_{0}^{\mathrm{c}}\right) \\
& =V_{N}\left(\phi_{0}^{\boldsymbol{u}^{\mathrm{c}}, \mathbf{0}}, \boldsymbol{u}^{\mathrm{c}}\right)-\ell\left(\phi_{j}^{\boldsymbol{u}^{\mathrm{mpc}}, \mathbf{0}}, u_{j}^{\mathrm{mpc}}\right) \\
& \leq V_{N}^{*}(x)-\sum_{i=0}^{j} \ell\left(\phi_{i}^{\boldsymbol{u}^{\mathrm{mpc}}, \mathbf{0}}, u_{i}^{\mathrm{mpc}}\right) .
\end{aligned}
$$

It follows from the optimality principle that $V_{N}^{*}\left(\phi_{j+1}^{\boldsymbol{u}^{\mathrm{mpc}}, \boldsymbol{w}}\right) \leq$ $V_{N}\left(\phi_{j+1}^{\boldsymbol{u}^{\mathrm{mpc}}, \boldsymbol{w}}, \hat{\boldsymbol{u}}^{\mathrm{c}}\right)$. This inequality along with (27) in turn implies that

$$
V_{N}^{*}\left(\phi_{j+1}^{\boldsymbol{u}^{\mathrm{mpc}}, \boldsymbol{w}}\right) \leq V_{N}^{*}(x)-\sum_{i=0}^{j} \ell\left(\phi_{i}^{\boldsymbol{u}^{\mathrm{mpc}}, \mathbf{0}}, u_{i}^{\mathrm{mpc}}\right) .
$$

5) Robust convergence: First, observe that (13) is an immediate consequence of (24) and (28). Let us now recall that $x=\xi_{\tau_{t}}$ and $\phi_{j+1}^{\boldsymbol{u}^{\mathrm{mpc}}, \boldsymbol{w}}(x)=\xi_{\tau_{t+1}}$. Then, one can rewrite (28) as follows:

$V_{N}^{*}\left(\xi_{\tau_{t+1}}\right)-V_{N}^{*}\left(\xi_{\tau_{t}}\right) \leq-\sum_{i=0}^{\tau_{t+1}-\tau_{t}-1} \ell\left(\phi_{i}^{\boldsymbol{u}^{\mathrm{mpc}}, \mathbf{0}}\left(\xi_{\tau}\right), u_{i}^{\mathrm{mpc}}\left(\xi_{\tau}\right)\right)$.

The right-hand side of the above inequality is strictly negative unless when $\phi_{i}^{\boldsymbol{u}^{\mathrm{mpc}}, \mathbf{0}}\left(\xi_{\tau}\right) \in \mathcal{T}_{i}^{\mathbb{X}}$ and $u_{i}^{\mathrm{mpc}}\left(\xi_{\tau}\right) \in \mathcal{T}_{i}^{\mathbb{U}}$ for all $i \in$ $\left\{0: \tau_{t+1}-\tau_{t}-1\right\}$. Since $V_{N}^{*}\left(\xi_{\tau_{t+1}}\right)$ is a nonnegative value, it is straightforward to observe that the states and inputs of the closed-loop dynamics (7) converge to their corresponding target sets. This concludes the proof.

\section{B. Proof of Theorems IV.4 and IV.5}

We first begin with a preliminary argument that is shared between both theorems. We then carry on with the proof of each case in an orderly fashion. Notice that $\xi \in \mathcal{S} \ominus M \mathcal{B}(l, u)$ and $\mathcal{S}$ is a polytope by the theorems' hypothesis. Let $h_{M \mathcal{B}}$ be the support function of $M \mathcal{B}$. One can infer that

$$
\left\langle a_{i, \mathcal{S}}^{\top}, \xi\right\rangle \leq b_{i, \mathcal{S}}-h_{M \mathcal{B}}\left(a_{i, \mathcal{S}}^{\top}\right), \forall i \in\{1: m\} .
$$

Next, observe that $\mathcal{B}(l, u) \subset \mathbb{R}^{k}$ is a polytope (and as a result bounded), and the domain $\mathcal{K}_{\mathcal{B}}$ on which the support function $h_{\mathcal{B}}$ is defined is the whole space, i.e., $\mathcal{K}_{\mathcal{B}}=\mathbb{R}^{k}$. Hence, $h_{M \mathcal{B}}\left(a_{i, \mathcal{S}}^{\top}\right)=h_{\mathcal{B}}\left(M^{\top} a_{i, \mathcal{S}}^{\top}\right)$, and as a consequence

$$
\left\langle a_{i, \mathcal{S}}^{\top}, \xi\right\rangle \leq b_{i, \mathcal{S}}-h_{\mathcal{B}}\left(M^{\top} a_{i, \mathcal{S}}^{\top}\right), \forall i \in\{1: m\} .
$$

Rearranging the above inequality, we arrive at

$$
h_{\mathcal{B}}\left(M^{\top} a_{i, \mathcal{S}}^{\top}\right) \leq b_{i, \mathcal{S}}-\left\langle a_{i, \mathcal{S}}^{\top}, \xi\right\rangle, \forall i \in\{1: m\}
$$

where the only unknown entity is $h_{\mathcal{B}}\left(M^{\top} a_{i, \mathcal{S}}^{\top}\right)$ with $M^{\top} a_{i, \mathcal{S}}^{\top} \in$ $\mathbb{R}^{k}$. It follows from the definition of the support function that $\left\langle M^{\top} a_{i, \mathcal{S}}^{\top}, z\right\rangle \leq h_{\mathcal{B}}\left(M^{\top} a_{i, \mathcal{S}}^{\top}\right)$ for all $z \in \mathbb{R}^{k}$. Thus

$$
\left\langle M^{\top} a_{i, \mathcal{S}}^{\top}, z\right\rangle \leq b_{i, \mathcal{S}}-\left\langle a_{i, \mathcal{S}}^{\top}, \xi\right\rangle, \forall i \in\{1: m\}, \forall z \in \mathcal{B} .
$$

Let us now define for all $i \in\{1: m\}, a_{i, \overline{\mathcal{S}}}^{\top}:=M^{\top} a_{i, \mathcal{S}}^{\top}, b_{i, \overline{\mathcal{S}}}:=$ $b_{i, \mathcal{S}}-\left\langle a_{i, \mathcal{S}}^{\top}, \xi\right\rangle$, and the convex polytope (which we referred to as the principal polytope in the paragraph before Theorem IV.4)

$$
\begin{aligned}
\overline{\mathcal{S}} & :=\left\{s \in \mathbb{R}^{k}:\left\langle a_{i, \overline{\mathcal{S}}}^{\top}, s\right\rangle \leq b_{i, \overline{\mathcal{S}}}, \forall i \in\{1: m\}\right\} \\
& =\left\{s \in \mathbb{R}^{k}: A_{\overline{\mathcal{S}}} s \leq b_{\overline{\mathcal{S}}}\right\}
\end{aligned}
$$

where $A_{\overline{\mathcal{S}}}:=\left[a_{1, \overline{\mathcal{S}}}^{\top}, \ldots, a_{m, \overline{\mathcal{S}}}^{\top}\right]^{\top}=\left(M^{\top} A_{\mathcal{S}}^{\top}\right)^{\top}=A_{\mathcal{S}} M \quad$ and $b_{\overline{\mathcal{S}}}:=\left[b_{1, \overline{\mathcal{S}}}, \ldots, b_{m, \overline{\mathcal{S}}}\right]^{\top}=b_{\mathcal{S}}-A_{\mathcal{S}} \xi$. Now, one can deduce from the inequalities (29) and the definition (30) that the convex polytope $\overline{\mathcal{S}}$ contains the hyperrectangle $\mathcal{B}(l, u)$, i.e., $\mathcal{B}(l, u) \subseteq \overline{\mathcal{S}}$. Notice that $\mathcal{B}(l, u)$ is parametric in the variables $l$ and $u$.

Theorem IV.4: In the CP framework, we propose a convex nonlinear program to compute the hyperrectangle $\mathcal{B}(l, u) \subseteq$ $\overline{\mathcal{S}}$ such that its volume is maximized. Suppose $\mathcal{B}(l, u)$ is parameterized as $l:=-\underline{v}=\left[-\underline{v}_{1}, \ldots,-\underline{v}_{k}\right]^{\top}$ and $u:=\bar{v}=$ $\left[\bar{v}_{1}, \ldots, \bar{v}_{k}\right]^{\top}$ such that for all $i \in\{1: k\}, \underline{v}_{i}$ and $\bar{v}_{i}$ are positive scalars (this condition has to do with the fact that the resulting hyperrectangle should contain the origin). Recall the inequality (29), that is $\left\langle M^{\top} a_{i, \mathcal{S}}^{\top}, z\right\rangle \leq b_{i, \mathcal{S}}-a_{i, \mathcal{S}} \xi$, for all $i \in\{1: m\}$ and for all $z \in \mathcal{B}$. In what follows, we show that although the hyperrectangle $\mathcal{B}(l, u)=\mathcal{B}(-\underline{v}, \bar{v})$ is parametric, one can provide a closed form for its support function evaluated at $M^{\top} a_{i, \mathcal{S}}^{\top}$. By definition of a support function

$$
\begin{aligned}
h_{\mathcal{B}}\left(M^{\top} a_{i, \mathcal{S}}^{\top}\right)=\max _{z}\left\langle M^{\top} a_{i, \mathcal{S}}^{\top}, z\right\rangle \\
\text { s.t. } \quad A_{\mathcal{B}} z \leq b_{\mathcal{B}}
\end{aligned}
$$

where $A_{\mathcal{B}}=\left[\mathrm{I}_{k}-\mathrm{I}_{k}\right]^{\top}$ and $b_{\mathcal{B}}=\left[\bar{v}^{\top} \underline{v}^{\top}\right]^{\top}$. The above problem is an LP with a bounded feasible set. Thus, the optimal solution lies on the boundary of the hyperrectangle toward which the normal $M^{\top} a_{i, \mathcal{S}}^{\top}$ points. Let us define, for all $i \in\{1: m\}$, $\hat{w}^{i}:=\operatorname{sign}\left(M^{\top} a_{i, \mathcal{S}}^{\top}\right) \in \mathbb{R}^{k}$, where the sign operator is applied entrywise. (Notice that this vector simply indicates the orthant(s) that the vector $M^{\top} a_{i, \mathcal{S}}^{\top}$ points to.) It then becomes clear that the 
vectors $w^{i} \in \mathbb{R}^{2 k}$, as defined in (16), enable us to express the optimal solution of (31) in terms of a linear combination of the vertices of $\mathcal{B}$, i.e.

$$
h_{\mathcal{B}}\left(M^{\top} a_{i, \mathcal{S}}^{\top}\right)=\left\langle w^{i},\left[\bar{v}^{\top} \underline{v}^{\top}\right]^{\top}\right\rangle, \forall i \in\{1: m\} .
$$

Based on the above relation, the inequality (29) simplifies to

$$
\left\langle w^{i},\left[\bar{v}^{\top} \underline{v}^{\top}\right]^{\top}\right\rangle \leq b_{i, \mathcal{S}}-a_{i, \mathcal{S}} \xi, \forall i \in\{1: m\}
$$

in which the vectors $\underline{v}, \bar{v} \in \mathbb{R}^{k}$ are the decision variables. Intuitively, the above inequalities represent the linear constraints that the vertices of the hyperrectangle $\mathcal{B}(-\underline{v}, \bar{v})$ should satisfy in order to guarantee $\xi \in \mathcal{S} \ominus M \mathcal{B}(-\underline{v}, \bar{v})$.

Based on the chosen definition of volume for $\mathcal{B}(-\underline{v}, \bar{v})$ in (11), we intend to find a hyperrectangle $\mathcal{B}(-\underline{v}, \bar{v})$ that possesses the maximal volume. Unfortunately, regardless of the definition choice for the volume, the resulting objective function is nonconvex and becomes unsuitable for optimization. Interestingly enough, one can simply use the logarithmic mapping for the volume definitions in (11) to obtain the objective functions suggested in (15), that are monotonic nonlinear concave functions. Then, it follows that a maximum hyperrectangle $\mathcal{B}$ that contains the origin and satisfies $\xi \in \mathcal{S} \ominus M \mathcal{B}$ is the solution of the CP (14).

Theorem IV.5: In the LP framework, we follow the procedure proposed in [37] with which one is able to cast the problem as a linear program. We first provide the proof for the LP relaxation of the problem (14) with $q=1$. Let us denote the maximum length of a line segment containing the origin, parallel to the $j$ th coordinate axis, and contained in $\overline{\mathcal{S}}$ by $r_{j}$. It follows from [37, Prop. 3] that one can use (17b) to find $r_{j}$, for all $j \in\{1: k\}$. It is worth noting that in the LP (17b), the constraints $z \leq 0$ and $z+\omega e_{j} \geq 0$ are two extra regularity conditions that we placed on the line segment compared to [37, Prop. 3]. These conditions ensure that the origin lies inside this line segment. Now, define the strictly positive vector $r \in \mathbb{R}^{k}$ by $r_{j}=\omega_{j}$ for all $j \in\{1: k\}$. Then, it follows from [37, Prop. 2] that a maximum $r$-constrained inner hyper-rectangular $\mathcal{B}$ of $\overline{\mathcal{S}}$ that contains the origin is given by $\mathcal{B}\left(z^{*}, z^{*}+\lambda^{*} r\right)$ where $z^{*}$ and $\lambda^{*}$ are the optimal solutions of (17a). Here, we also emphasize the fact that we have introduced the extra constraints $z \leq 0$ and $z+\lambda r \geq 0$ with respect to [37, Prop. 2]. By doing so, the LP (17a) is forced to find a hyperrectangular $\mathcal{B}$ such that it contains the origin. Then, the claim for the LP case follows.

We now present a sketch of proof for the LP relaxation of the problem (14) with $q=2$. Observe that the polytope $\overline{\mathcal{S}}^{\prime}:=\left\{s \in \mathbb{R}^{2 k}: W^{\prime} s \leq B^{\prime}\right\}$ is the inequality representation of the constraints in the $\mathrm{CP}(14)$, where $W^{\prime}$ and $B^{\prime}$ are defined in Theorem IV.5. We seek to find a hyperrectangle that fits inside this lifted polytope as follows. In the first step, we place a vertex of the hyperrectangle at the origin. We then find the width of the line segment along each coordinate that is inside the lifted polytope and contains the origin using (18b). In the second step, we use (18a) to find a scaling factor $\lambda$ such that the $\lambda$-scaled hyperrectangle constructed based on the first step fits inside the polytope $\overline{\mathcal{S}}^{\prime}$. This concludes the proof.
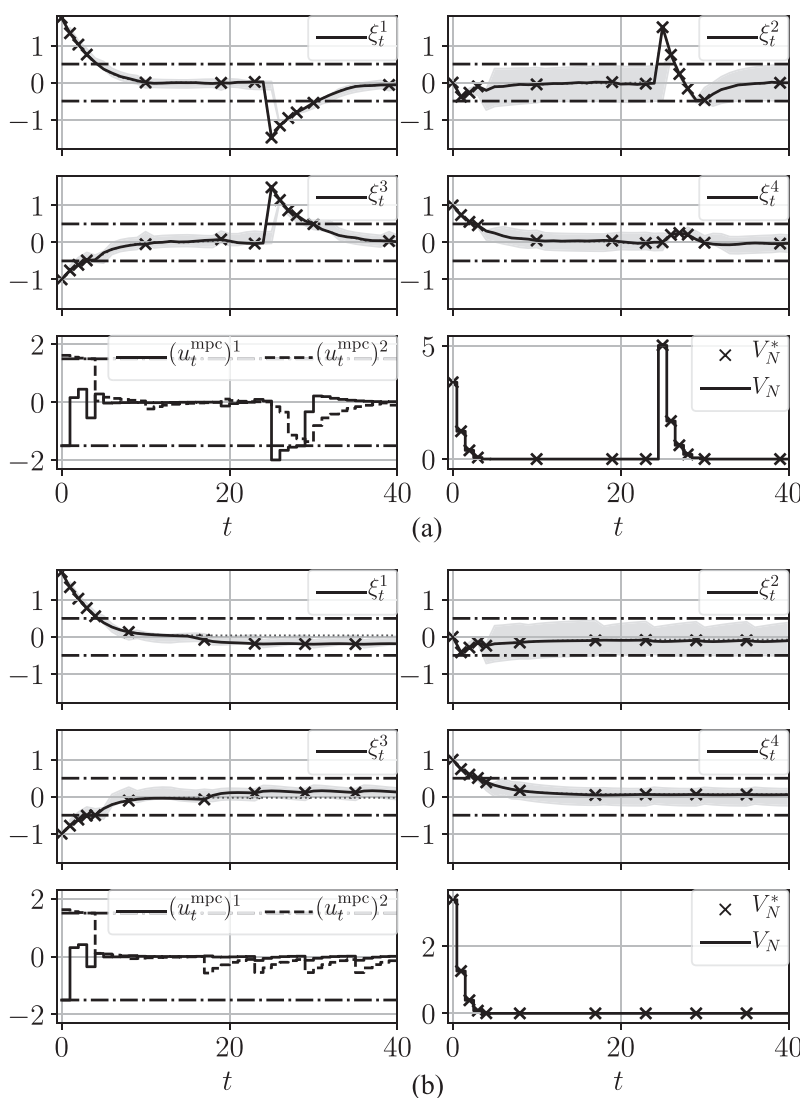

Fig. 2. Comparison of the event-based implementation using the construction approach $\mathrm{CP}_{1}$ with the standard implementation. (Top four) The solid lines are the evolution of states. The crosses are the states at triggering instances. The (gray) shaded areas are the projection of constructed hyperrectangles $\mathcal{E}$ on the corresponding state's coordinate axis. (Bottom left) The lines are the input of the closed-loop system. (Bottom right) The crosses are the value function $V_{N}^{*}$ at triggering instances. The solid line is the interevent cost function $V_{N}^{N}$ computed using Theorem IV.1. (a) Uniform disturbance. (b) Worst case disturbance.

\section{Numerical EXAMPLes}

In this section, we provide a numerical exam ple to study the results presented in Section IV. For the numerical simulations, we use CVXOPT [38] and (py)cddlib [39]. The system is an unstable batch reactor borrowed from [40, p. 213]. We discretized the model using the zero-order-hold method with step size 0.05 , that is

$$
\begin{aligned}
x^{+}= & \left(\begin{array}{cccc}
1.08 & -0.05 & 0.29 & -0.24 \\
-0.03 & 0.81 & 0.00 & 0.03 \\
0.04 & 0.19 & 0.73 & 0.24 \\
0.00 & 0.19 & 0.05 & 0.91
\end{array}\right) x \\
& +\left(\begin{array}{cc}
0.00 & -0.02 \\
0.26 & 0.00 \\
0.08 & -0.13 \\
0.08 & -0.00
\end{array}\right) u+w
\end{aligned}
$$



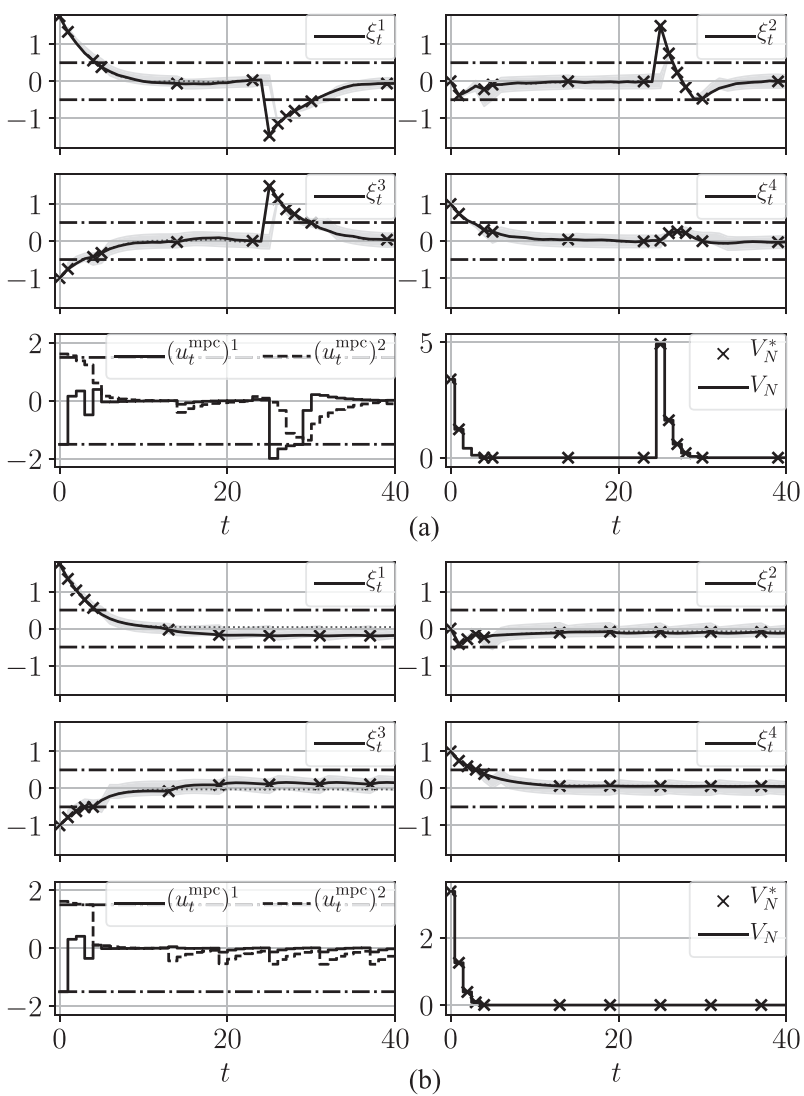

(b)

Fig. 3. Comparison of the event-based implementation using the construction approach $\mathrm{LP}_{1}$ with the standard implementation. (Top four) The solid lines are the evolution of states. The crosses are the states at triggering instances. The (gray) shaded areas are the projection of constructed hyperrectangles $\mathcal{E}$ on the corresponding state's coordinate axis. (Bottom left) The lines are the input of the closed-loop system. (Bottom right) The crosses are the value function $V_{N}^{*}$ at triggering instances. The solid line is the interevent cost function $V_{N}$ computed using Theorem IV.1. (a) Uniform disturbance. (b) Worst case disturbance.

where the state and input constraint sets are $\mathbb{X}=\left\{x \in \mathbb{R}^{4}\right.$ : $\left.\|x\|_{\infty} \leq 2\right\}$ and $\mathbb{U}=\left\{u \in \mathbb{R}^{2}:\|u\|_{\infty} \leq 2\right\}$, respectively. The disturbance set is defined as $\mathbb{W}=\left\{w \in \mathbb{R}^{4}:\|w\|_{\infty} \leq 0.02\right\}$. The state and input target sets are $\mathbb{T}_{x}=\left\{x \in \mathbb{R}^{4}:\|x\|_{\infty} \leq\right.$ $0.5\}$ and $\mathbb{T}_{u}=\left\{u \in \mathbb{R}^{2}:\|u\|_{\infty} \leq 1.5\right\}$, respectively. The horizon length $N$ is set to 10 . The weight matrices in the cost function (4) are $Q=2 \times \mathrm{I}_{4}$ and $r=\mathrm{I}_{2}$. Finally, the terminal set is $\mathcal{X}_{f}=\left\{x \in \mathbb{R}^{4}:\|x\|_{\infty} \leq 0.2\right\}$.

In what follows, we employ the triggering set construction approaches of Theorems IV.4 and IV.5 for $q=1$. Two types of disturbance realizations are considered: 1) a uniform distribution with the bounded support $\mathbb{W}$ and 2) a worst case disturbance $w_{t}=\operatorname{argmax}_{w \in \mathbb{W}} \xi_{t}^{\top} w$ at each time $t$. In the case of uniform disturbance, we also manually applied an impulsetype disturbance to the closed-loop dynamics by resetting the second state $\xi_{25}^{2}$ to 1.7 . This disturbance does not belong to the admissible disturbance set $\mathbb{W}$.

Figs. 2 and 3 show the behavior of the event-based implementation of the MPC method. (Notice that the behavior of the standard MPC was almost identical, we did not include the results of the standard MPC for the sake of clarity.)

We begin by pointing out the shared properties of the approaches $\mathrm{CP}_{1}$ and $\mathrm{LP}_{1}$. First of all, it is evident that the number of instances that the optimization problem (5) is solved has effectively reduced in all considered cases compared to standard periodic implementation. Observe that the inputs and states of the closed-loop dynamics (7) do not violate the constraint sets $\mathbb{X}$ and $\mathbb{U}$, respectively, in all considered cases. Moreover, the closed-loop states $\xi_{t}$ and the inputs $u_{t}$ converge to the target sets $\mathbb{T}_{x}$ and $\mathbb{T}_{u}$, respectively. Finally, both of the approaches $\mathrm{CP}_{1}$ and $\mathrm{LP}_{1}$ can effectively recover from the impulse-type disturbance applied on time $t=25$. We also note that the event-based implementations exhibit an almost limit-cyclic behavior inside the target set $\mathbb{T}_{x}$ in the worst case disturbance realizations.

Let us now highlight the difference between the construction approaches $\mathrm{CP}_{1}$ and $\mathrm{LP}_{1}$. As depicted in the top right plots of Figs. 2(a) and 3(a), the construction method $\mathrm{LP}_{1}$ is more conservative in comparison with the construction method $\mathrm{CP}_{1}$. The width of the shaded areas represents the projection of the triggering sets $\mathcal{E}(x)$. In Fig. 2(a), one can also observe in the top right plot that the triggering intervals are tight with respect to the target sets as well.

\section{FUtURE DiRECTIONS}

In this paper, an ET approach was proposed to implement an RMPC method to constrained, perturbed LTI systems. The procedure to design the triggering mechanism is online, and is decoupled from the controller design. Specifically, we introduced two theoretical frameworks to construct the triggering mechanism as a volume maximization problem. There are multiple directions that one can pursue to extend the results in this paper. First, it is interesting to investigate the possibility of extending the results of this paper to a nonlinear MPC case. In a qualitative manner, we have observed that the choice of tightening gains $\boldsymbol{K}$ directly impacts the constructed triggering sets $\mathcal{E}$. Hence, another possible direction is to explore the possibility of characterizing this unknown dependency in a more quantitative manner. Finally, the triggering approach proposed in this paper is online (and in fact, state dependent). It is thus valuable to investigate whether it is possible to make the triggering set design offline.

\section{REFERENCES}

[1] J. Baillieul and P. J. Antsaklis, "Control and communication challenges in networked real-time systems," Proc. IEEE, vol. 95, no. 1, pp. 9-28, Jan. 2007.

[2] W. P. M. H. Heemels, K. H. Johansson, and P. Tabuada, "An introduction to event-triggered and self-triggered control," in Proc. 51st IEEE Conf. Decis. Control, 2012, pp. 3270-3285.

[3] P. Tabuada, "Event-triggered real-time scheduling of stabilizing control tasks," IEEE Trans. Autom. Control, vol. 52, no. 9, pp. 1680-1685, Sep. 2007.

[4] A. Girard, "Dynamic triggering mechanisms for event-triggered control,' IEEE Trans. Autom. Control, vol. 60, no. 7, pp. 1992-1997, Jul. 2015.

[5] A. Anta and P. Tabuada, "To sample or not to sample: Self-triggered control for nonlinear systems," IEEE Trans. Autom. Control, vol. 55, no. 9, pp. 2030-2042, Sep. 2010. 
[6] C. Nowzari and J. Cortés, "Self-triggered coordination of robotic networks for optimal deployment," Automatica, vol. 48, no. 6, pp. 1077-1087, 2012.

[7] E. F. Camacho and C. B. Alba, Model Predictive Control. London, U.K.: Springer-Verlag, 2013.

[8] D. L. Marruedo, T. Álamo, and E. F. Camacho, "Input-to-state stable MPC for constrained discrete-time nonlinear systems with bounded additive uncertainties," in Proc. 41st IEEE Conf. Decis. Control, 2002, vol. 4 pp. 4619-4624.

[9] D. Q. Mayne, J. B. Rawlings, C. V. Rao, and P. O. M. Scokaert, "Constrained model predictive control: Stability and optimality," Automatica, vol. 36, no. 6, pp. 789-814, 2000.

[10] D. Q. Mayne, "Model predictive control: Recent developments and future promise," Automatica, vol. 50, no. 12, pp. 2967-2986, 2014

[11] E. A. Yildirim and S. J. Wright, "Warm-start strategies in interior-point methods for linear programming," SIAM J. Optim., vol. 12, no. 3, pp. 782 $810,2002$.

[12] A. Willig, "Recent and emerging topics in wireless industrial communications: A selection,” IEEE Trans. Ind. Inform., vol. 4, no. 2, pp. 102-124, May 2008.

[13] L. Krishnamachari, D. Estrin, and S. Wicker, "The impact of data aggregation in wireless sensor networks," in Proc. 22nd Int. Conf. Distrib. Comput. Syst. Workshops, 2002, pp. 575-578.

[14] S. Madden, M. J. Franklin, J. M. Hellerstein, and W. Hong, "TAG: A tiny aggregation service for ad-hoc sensor networks," ACM SIGOPS Oper. Syst. Rev., vol. 36, no. SI, pp. 131-146, 2002.

[15] A. Richards and J. P. How, "Robust stable model predictive control with constraint tightening," in Proc. Amer. Control Conf., 2006 pp. 15571562 .

[16] O. A. Z. Sotomayor and C. Garcia, "Model-based predictive control of a pre-denitrification plant: A linear state-space model approach," IFAC Proc. Vol., vol. 35, no. 1, pp. 429-434, 2002.

[17] A. Kelman, Y. Ma, and F. Borrelli, "Analysis of local optima in predictive control for energy efficient buildings," J. Building Perform. Simul., vol. 6 , no. 3, pp. 236-255, 2013.

[18] T. G. Hovgaard, S. Boyd, L. F. Larsen, and J. B. Jørgensen, "Nonconvex model predictive control for commercial refrigeration," Int. J. Control, vol. 86, no. 8, pp. 1349-1366, 2013

[19] S. Bregman, A. S. Kolarijani, and T. Keviczky, "Robust model predictive control with aperiodic actuation," in Proc. 56th IEEE Conf. Decis. Control, 2017, pp. 5457-5462.

[20] G. C. Goodwin, H. Haimovich, D. E. Quevedo, and J. S. Welsh, "A moving horizon approach to networked control system design," IEEE Trans. Autom. Control, vol. 49, no. 9, pp. 1427-1445, Sep. 2004

[21] C. V. Rao, J. B. Rawlings, and D. Q. Mayne, "Constrained state estimation for nonlinear discrete-time systems: Stability and moving horizon approximations," IEEE Trans. Autom. Control, vol. 48, no. 2, pp. 246-258, Feb. 2003

[22] J. Sijs, M. Lazar, and W. P. M. H. Heemels, "On integration of event-based estimation and robust MPC in a feedback loop," in Proc. 13th ACM Int. Conf. Hybrid Syst., Comput. Control, 2010, pp. 31-40.

[23] D. Bernardini and A. Bemporad, "Energy-aware robust model predictive control based on noisy wireless sensors," Automatica, vol. 48, no. 1, pp. 36-44, 2012.

[24] A. Bemporad, M. Morari, V. Dua, and E. N. Pistikopoulos, "The explicit linear quadratic regulator for constrained systems," Automatica, vol. 38, no. 1, pp. 3-20, 2002.

[25] D. Lehmann, E. Henriksson, and K. H. Johansson, "Event-triggered mode predictive control of discrete-time linear systems subject to disturbances," in Proc. Eur. Control Conf., 2013, pp. 1156-1161.

[26] D. Antunes and W. P. M. H. Heemels, "Rollout event-triggered control: Beyond periodic control performance," IEEE Trans. Autom. Control, vol. 59, no. 12 , pp. 3296-3311, Dec. 2014.

[27] D. P. Bertsekas, Dynamic Programming and Optimal Control, vol. 1, 4th ed. Belmont, MA, USA: Athena Scientific, 2017

[28] F. D. Brunner, W. P. M. H. Heemels, and F. Allgöwer, "Robust eventtriggered MPC with guaranteed asymptotic bound and average sampling rate," IEEE Trans. Autom. Control, vol. 62, no. 11, pp. 5694-5709, Nov. 2017.

[29] S. V. Raković, B. Kouvaritakis, R. Findeisen, and M. Cannon, "Homothetic tube model predictive control," Automatica, vol. 48, no. 8, pp. 1631-1638, 2012 .
[30] B. Demirel, E. Ghadimi, D. E. Quevedo, and M. Johansson, "Optimal control of linear systems with limited control actions: Threshold-based event-triggered control," IEEE Trans. Control Netw. Syst., vol. 5, no. 3, pp. 1275-1286, Sep. 2017

[31] G. P. Incremona, A. Ferrara, and L. Magni, "Asynchronous networked MPC with ISM for uncertain nonlinear systems," IEEE Trans. Autom. Control, vol. 62, no. 9, pp. 4305-4317, Sep. 2017.

[32] V. Utkin and J. Shi, "Integral sliding mode in systems operating under uncertainty conditions," in Proc. 35th IEEE Conf. Decis. Control, 1996, vol. 4, pp. 4591-4596.

[33] J. C. Dunn and D. P. Bertsekas, "Efficient dynamic programming implementations of Newton's method for unconstrained optimal control problems," J. Optim. Theory Appl., vol. 63, no. 1, pp. 23-38, 1989.

[34] Y. Wang and S. Boyd, "Fast model predictive control using online optimization," IEEE Trans. Control Syst. Technol., vol. 18, no. 2, pp. 267-278, Mar. 2010.

[35] S. Richter, C. N. Jones, and M. Morari, "Computational complexity certification for real-time MPC with input constraints based on the fast gradien method," IEEE Trans. Autom. Control, vol. 57, no. 6, pp. 1391-1403, Jun. 2012.

[36] I. Kolmanovsky and E. G. Gilbert, "Theory and computation of disturbance invariant sets for discrete-time linear systems," Math. Problems Eng., vol. 4, no. 4, pp. 317-367, 1998.

[37] A. Bemporad, C. Filippi, and F. D. Torrisi, "Inner and outer approximations of polytopes using boxes," Comput. Geometry, vol. 27, no. 2, pp. 151-178, 2004

[38] M. Andersen, J. Dahl, and L. Vandenberghe, "CVXOPT: A Python package for convex optimization, version 1.1.6," 2013

[39] K. Fukuda, "cddlib reference manual, cddlib version 0.92," ETHZ, Zürich, Switzerland, 2001.

[40] H. H. Rosenbrock, Computer Aided Control System Design. New York, NY, USA: Academic, 1974.

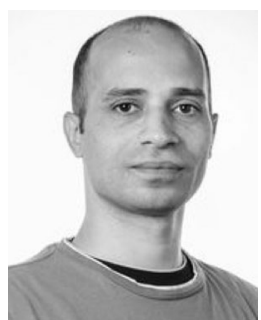

Arman Sharifi Kolarijani received the M.Sc. degree in applied mechanics from the Sharif University of Technology, Tehran, Iran, in 2011, and the M.Sc. and Ph.D. degrees in systems and control from the Delft University of Technology, Delft, The Netherlands, in 2014 and 2019, respectively.

$\mathrm{He}$ is currently a Postdoctoral Researcher with the Delft University of Technology. From 2008 to 2012, he was an Associate Researcher with Actuators Lab, Khaj-e-Nasir University of Technology, Tehran, Iran, working on the design and construction of rehabilitation device for lower limbs. His current research interests include optimization algorithms, optimal control, and symbolic verification of networked control systems.

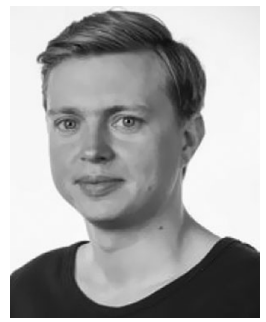

Sander Christian Bregman received the M.Sc. degree in systems and control from the Delft University of Technology, Delft, The Netherlands.

$\mathrm{He}$ is currently a Teacher with the Delft Center for Systems and Control. His interests range from optimization methods and model predictive control to more practical sides of control engineering. 


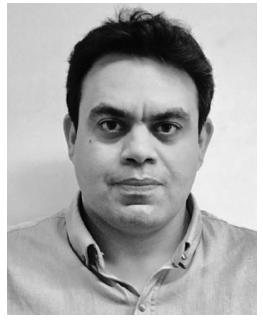

Peyman Mohajerin Esfahani received the B.Sc. and M.Sc. degrees from Sharif University of Technology, Tehran, Iran, in 2005 and 2007, respectively and the Ph.D. degree from ETH Zürich, Zürich, Switzerland, in 2014.

$\mathrm{He}$ is currently an Assistant Professor with the Delft Center for Systems and Control, Delft University of Technology, Delft, The Netherlands. Prior to joining TU Delft, he held several research appointments with EPFL, ETH Zurich, and MIT between 2014 and 2016. His research interests include theoretical and practical aspects of decision-making problems in uncertain and dynamic environments, with applications to control and security of large-scale and distributed systems.

Dr. Esfahani was one of the three finalists for the Young Researcher Prize in Continuous Optimization awarded by the Mathematical Optimization Society in 2016, and was a recipient of the 2016 George S. Axelby Outstanding Paper Award from the IEEE Control Systems Society.

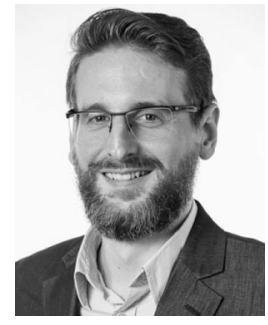

Tamás Keviczky received the M.Sc. degree in electrical engineering from the Budapest University of Technology and Economics, Budapest, Hungary, in 2001, and the Ph.D. degree from the Control Science and Dynamical Systems Center, University of Minnesota, Minneapolis, MN, USA, in 2005.

$\mathrm{He}$ is currently an Associate Professor with the Delft Center for Systems and Control, Delft University of Technology, Delft, The Netherlands. He was a Postdoctoral Scholar in Control and Dynamical Systems, California Institute of Technology, Pasadena CA, USA. His research interests include distributed optimization and optimal control, model predictive control, embedded optimization-based control, and estimation of large-scale systems with applications in aerospace, automotive, mobile robotics, industrial processes, and infrastructure systems, such as water, heat, and power networks.

Dr. Keviczky was a corecipient of the AACC O. Hugo Schuck Best Paper Award for Practice in 2005. He was an Associate Editor for Automatica between 2011 and 2017. 